\title{
MOST observations of the young open cluster NGC 2264^
}

\author{
K. Zwintz ${ }^{1}$, M. Hareter ${ }^{1}$, R. Kuschnig ${ }^{2,1}$, P. J. Amado ${ }^{3}$, N. Nesvacil ${ }^{1}$, E. Rodriguez ${ }^{3}$, D. Diaz-Fraile ${ }^{3}$, W. W. Weiss ${ }^{1}$,
} T. Pribulla ${ }^{4,5}$, D. B. Guenther ${ }^{6}$, J. M. Matthews ${ }^{2}$, A. F. J. Moffat ${ }^{7}$, S. M. Rucinski ${ }^{8}$, D. Sasselov ${ }^{9}$, and G. A. H. Walker ${ }^{2}$

1 Institut für Astronomie, Universität Wien, Türkenschanzstrasse 17, 1180 Vienna, Austria e-mail: zwintz@astro.univie.ac.at

2 Department of Physics and Astronomy, University of British Columbia, 6224 Agricultural Road, Vancouver, BC V6T 1Z1, Canada e-mail: [kuschnig; matthews]@astro.ubc.ca; gordonwa@uvic.ca

3 Instituto de Astrofísica de Andalucía (CSIC), PO Box 3004, 18080 Granada, Spain e-mail: [pja; eloy; dario]@iaa.es

4 Astrophysikalisches Institut und Universitäts-Sternwarte, Schillergässchen 2-3, 07740 Jena, Germany e-mail: pribulla@ta3.sk

5 Astronomical Institute of the Slovak Academy of Sciences, 05960 Tatranská Lomnica, The Slovak Republic

${ }^{6}$ Department of Astronomy and Physics, St. Mary's University, Halifax, NS B3H 3C3, Canada e-mail: guenther@ap.smu.ca

7 Départment de physique, Université de Montréal, C.P. 6128, Succ. Centre-Ville, Montréal, QC H3C 3J7, Canada e-mail: moffat@astro.umontreal.ca

8 David Dunlap Observatory, University of Toronto, PO Box 360, Richmond Hill, ON L4C 4Y6, Canada e-mail: rucinski@astro.utoronto.ca

9 Harvard-Smithsonian Center for Astrophysics, 60 Garden Street, Cambridge, MA 02138, USA e-mail: sasselov@cfa.harvard.edu

Received 17 February 2009 / Accepted 5 May 2009

\begin{abstract}
Context. Photometric time series obtained from space are one of the best ways to study pulsations in pre-main sequence (PMS) stars, especially as the corresponding amplitudes are at the millimagnitude level or below. We present high-precision time-series photometry of stars in the field of NGC 2264 obtained with the MOST (Microvariability \& Oscillations of STars) satellite.

Aims. A search for pulsating A and F type members of NGC 2264 with ultra-precise MOST photometry was conducted.

Methods. 68 stars in the field of NGC 2264 were observed simultaneously with the MOST satellite, 34 of which were previously identified as potential targets to search for PMS pulsation. The routines SIGSPEC and Period04 were used for the frequency analysis. Results. We discovered pulsation in 4 potential A and F type PMS members of NGC 2264 with frequencies between $2.7 \mathrm{~h}$ and $23 \mathrm{~min}$. For one of the PMS pulsators identified with MOST after the first days of observation, simultaneous ground-based Strömgren photometry was obtained with the OSN $0.9 \mathrm{~m}$ telescope and confirms the highest frequency identified in the MOST photometry. 26 stars with other (i.e., hotter and cooler) spectral types show clear variability: SPB and $\gamma$ Doradus pulsation, red giant oscillations, eclipsing binaries and variability caused by rotational modulation.
\end{abstract}

Key words. stars: pre-main sequence - open clusters and associations: individual: NGC $2264-\delta$ Sct - techniques: photometric

\section{Introduction}

The very first stages in the lives of stars remain obscured from view as protostars are completely buried in the molecular cloud from which they are born. Their further evolution strongly depends on their masses. Low to intermediate mass stars (i.e. with masses below $\sim 8 M_{\odot}$ ) have a pre-main sequence (PMS) phase, in which they gain their energy from gravitational contraction. Stars of higher masses burn hydrogen already when they first become optically visible, i.e., the birthline intersects the zero-age main sequence (ZAMS) for stars more massive than $\sim 8 M_{\odot}$ (Palla \& Stahler 1990 and 1993). Low mass PMS

\footnotetext{
* Based on data from the MOST satellite, a Canadian Space Agency mission, jointly operated by Dynacon Inc., the University of Toronto Institute for Aerospace Studies and the University of British Columbia with the assistance of the University of Vienna.
}

stars $\left(M<1.5 M_{\odot}\right)$ presumably evolve to T Tauri stars (Joy 1942) with spectral types from late $F$ to $M$. Intermediate mass PMS stars $\left(1.5 M_{\odot}<M<8 M_{\odot}\right)$ develop more rapidly to Herbig Ae/Be (HAEBE) objects (e.g., Herbig 1960; Finkenzeller \& Mundt 1984) with spectral types B to early F. The interaction of PMS stars with the remnants of their birth clouds induces typical observational features such as emission lines, infrared and/or ultraviolet excesses and (ir)regular brightness variations over a wide range of time scales and amplitudes.

Close to the ZAMS, the evolutionary tracks for pre- and post-main sequence stars intersect several times (e.g., Breger \& Pamyatnykh 1998). Therefore at a given combination of effective temperature $\left(T_{\text {eff }}\right)$, luminosity $\left(L / L_{\odot}\right)$ and mass $\left(M_{\odot}\right)$ the evolutionary phase of a field star is ambiguous and cannot be derived only from the respective evolutionary tracks. Similar envelope properties of pre- and post-main sequence objects are the 
cause for this ambiguity (Marconi \& Palla 1998). The differences, though, lie in the interior structures: PMS stars have a quite homologous core with a uniform distribution of density and chemical compositon, while post-main sequence stars are characterized by strong chemical inhomogeneities and the presence of nuclear reactions.

On their way to the ZAMS, PMS stars cross the instability region and can become pulsationally unstable. Their pulsations are driven by the same opacity mechanisms which occur in the more evolved classical $\delta$ Scuti stars, namely the $\kappa$ and/or $\gamma$ mechanisms in the $\mathrm{H}$ and $\mathrm{He}$ ionization zones (Marconi \& Palla 1998). As the pulsational properties depend on the inner structures of stars, asteroseismology can be used to test the evolutionary phase of a given star. The frequency spectra of $\ell=0$ modes of same radial order are nearly identical for pre- and post-main sequence pulsators (Suran et al. 2001). But for nonradial modes $(\ell>0)$ the patterns differ significantly due to the evolutionary changes in the stellar interiors and allow one to distinguish between the two evolutionary stages (Guenther et al. 2007).

Young open clusters provide an ideal testing ground for asteroseismic models, as the ages of member stars can be derived from the cluster properties independently. Clusters that are younger than 10 million years only have a short main sequence consisting of $\mathrm{O}$ and $\mathrm{B}$ type stars, while stars of later spectral types are still contracting towards the ZAMS. Within the last few years, pulsations have been discovered in $18 \mathrm{~A}$ and $\mathrm{F}$ type PMS members of 6 young clusters (e.g. Zwintz \& Weiss 2006; Zwintz et al. 2005).

In this paper we present space-based observations of the young open cluster NGC 2264 obtained with the MOST satellite.

\section{The young open cluster NGC 2264}

Numerous studies have been devoted to the young open cluster NGC $2264\left(\alpha_{2000}=6^{\mathrm{h}} 41^{\mathrm{m}}, \delta_{2000}=+9^{\circ} 53^{\prime}\right)$. It has a diameter of $\sim 39$ arcmin, belongs to the Mon OB 1 association and is located about $30 \mathrm{pc}$ above the galactic plane.

The determination of the cluster's age and distance is relatively inaccurate: first, its main sequence consists only of the massive $\mathrm{O}$ and $\mathrm{B}$ stars, while stars of later spectral types are still in their PMS evolutionary phase; second, star formation might be still going on in NGC 2264. Different values for the age of the cluster are reported in the literature ranging from $3 \times$ $10^{6}$ years (e.g., Walker 1956; Sung et al. 2004) to $\sim 10^{7}$ years (Sagar et al. 1986). Some cluster members might even be only $0.15 \times 10^{6}$ years old, if stars are still being formed (Sung et al. 1997). A distance modulus of $9.4 \pm 0.25$ corresponding to a distance of $760 \pm 80 \mathrm{pc}$ was found by Sung et al. (1997). These values are consistent with those reported by other authors (e.g., Pérez et al. 1987).

NGC 2264 is not significantly affected by reddening and differential reddening is negligible as well. The mean value of $E(B-V)=0.071 \pm 0.033$ mag reported by Sung et al. (1997) is in good agreement with values found by other authors (e.g., Walker 1956; Park et al. 2000).

NGC 2264 hosts the very first ever discovered PMS pulsators, V 588 Mon and V 589 Mon (Breger 1972). While searching for $\delta$ Scuti stars in young clusters, he obtained photometric time series of the A and F type stars in NGC 2264 with a time base of only three nights distributed over two years. He determined the periods for V 588 Mon and V 589 Mon to be in the $\delta$ Scuti regime with 2.6 and $2.9 \mathrm{~h}$, respectively. Taking into account the young cluster age and the very likely membership of the two stars to the cluster, he concluded that V 588 Mon and V 589 Mon must be in their PMS evolutionary phase.

Recent studies were devoted to a better determination of the pulsation periods and amplitudes in V 588 Mon and V 589 Mon and to a detailed asteroseismic interpretation. Kallinger et al. (2008a) found 16 and 20 pulsation frequencies for V 588 Mon and V 589 Mon, respectively, based on observations obtained during a ground based multi-site campaign. The two PMS pulsators were also observed repeatedly using the MOST satellite (Kallinger et al. 2009), where many more pulsation frequencies were identified and studied asteroseismologically.

The aim of the NGC 2264 observations using the MOST satellite was the search for additional, previously undetected pulsating PMS cluster members at much lower noise levels than it was possible before.

\section{MOST observations}

The MOST (Microvariability and Oscillations of STars) space telescope (Walker et al. 2003) was launched on June 30, 2003, into a polar sun-synchronous circular orbit with an orbital period of $\sim 101 \mathrm{~min}$ (corresponding to an orbital frequency of $14.2 \mathrm{c} / \mathrm{d}$ ). From its orbital vantage point, MOST can obtain uninterrupted observations of stars located in its Continuous Viewing Zone (CVZ) for up to 8 weeks. The MOST satellite houses a $15-\mathrm{cm}$ Rumak-Maksutov telescope feeding a CCD photometer through a single, custom broadband optical filter (covering wavelengths from 350 to $750 \mathrm{~nm}$ ).

MOST can supply up to three types of photometric data simultaneously for multiple targets in its field. The mission was originally intended only for Fabry Imaging, in which an image of the entrance pupil of the telescope - illuminated by a bright target star $(V<6)$ - is projected onto the instrument's Science CCD by a Fabry microlens (see Reegen et al. 2006, for details). After MOST was operating in orbit, the pointing performance of the satellite was improved so much that a new mode of observing, Direct Imaging, was made practical. Direct Imaging is much like conventional CCD photometry, in which photometry is obtained from defocussed images of stars in the open area of the CCD not covered by the Fabry microlens array field stop mask. In the original mission, no scientific information was available from the Guide Stars used for the ACS (Attitude Control System), but now precise photometry is possible for these stars as well (see, e.g., Walker et al. 2005; Aerts et al. 2006).

Due to the magnitude range $(7<V<12 \mathrm{mag})$ and the large number of targets, the cluster was observed in the open field of the MOST Science CCD in Guide Star Photometry Mode. But as not all of the candidate PMS pulsators (i.e., A and F type cluster stars) could be reached using a single pointing of the satellite, two fields of observations were chosen (hereafter named "field A" and "field B") and observed in alternating halves of each 101-min orbit. On-board exposures are $1.5 \mathrm{~s}$ long (to satisfy the cadence of Guide Star ACS operations), but 16 consecutive exposures are "stacked" on board, and sampled about 2 times per minute.

Using this setting, MOST time series photometry was obtained for a total of 68 stars in the region of NGC 2264, where 38 stars were observed in field A and 30 in field B. Among all 68 stars, 32 objects have spectral types ranging from early A to mid F (23 in field A and 9 in field B) being ideal candidates to search for PMS pulsation. MOST observed NGC 2264 from December 7, 2006, to January 3, 2007. The light curves used for the analysis have time bases of $\sim 22.72$ days for field $\mathrm{A}$ and 
Table 1. Log of the simultaneous ground-based observations of HD 261387 using the OSN 0.9 m telescope and Strömgren $u v b y$ filters.

\begin{tabular}{ccr}
\hline \hline Date & Timebase [h] & Points \\
\hline & December 2006 & \\
$15-16$ & 2.9 & 32 \\
$16-17$ & 3.9 & 37 \\
$17-18$ & 2.6 & 31 \\
$18-19$ & 2.9 & 28 \\
$19-20$ & 3.3 & 28 \\
$20-21$ & 2.5 & 26 \\
$21-22$ & 3.3 & 44 \\
\hline & January 2007 & \\
$09-10$ & 2.1 & 32 \\
$11-12$ & 5.5 & 33 \\
$12-13$ & 5.7 & 79 \\
$16-17$ & 6.2 & 42 \\
$17-18$ & 6.1 & 90 \\
$18-19$ & 5.7 & 89 \\
$19-20$ & 6.8 & 80 \\
$20-21$ & 7.7 & 105 \\
\hline
\end{tabular}

22.77 days for field B. The effective duty cycles for field A and B are $18 \%$ and $29 \%$, respectively, and correspond to the fraction of each 101-min orbit during which observations were taken. The reason for this difference is that during each orbit field $\mathrm{B}$ was observed in better stray light conditions than field A.

\subsection{Simultaneous ground-based Strömgren photometry}

The $0.9 \mathrm{~m}$ telescope of the Observatorio de Sierra Nevada (OSN) in Granada, Spain, equipped with a 4-channel Strömgren photoelectric photometer, was used to conduct a simultaneous monitoring program for HD 261387 - one of the variables identified with MOST already during the first days of observation.

HD 261387 was observed with $90 \mathrm{~s}$ integrations for a few hours every night. HD 47469, HD 261585 and HD 47554 were used as comparison and check stars and sky measurements were taken according to the brightness of the Moon. The observations were conducted between December 15 and 21, 2006, and between January 9 and 20, 2007. Only four of those nights were lost due to bad weather. Data for HD 261387 was obtained in 15 out of 19 nights for a total amount of $67 \mathrm{~h}$ and 776 data points. The observing log for the simultaneous ground-based observations is given in Table 1 and the results are discussed in Sect. 6.3.

\section{Data reduction and frequency analysis}

The NGC 2264 data discussed in this paper were obtained exclusively in the MOST Guide Star photometry mode. For this type of MOST data, a special reduction method was developed by one of us (MH). It uses a similar approach as for targets observed in Fabry Imaging mode (Reegen et al. 2006), i.e., resolving linear correlations between the intensity of target and background pixels. Since MOST Guide Star photometry does not provide information on the intensity of the background, the correlations between constant and variable stars are used instead. In a first step, the MOST Guide Stars are classified into variable or constant objects by a quick-look analysis. The data of all selected intrinsically constant objects are then combined to a comparison light curve.

Stray light effects are corrected by subtraction of linear correlations between target and comparison time series. Selfevidently, comparison time series are assumed and required to

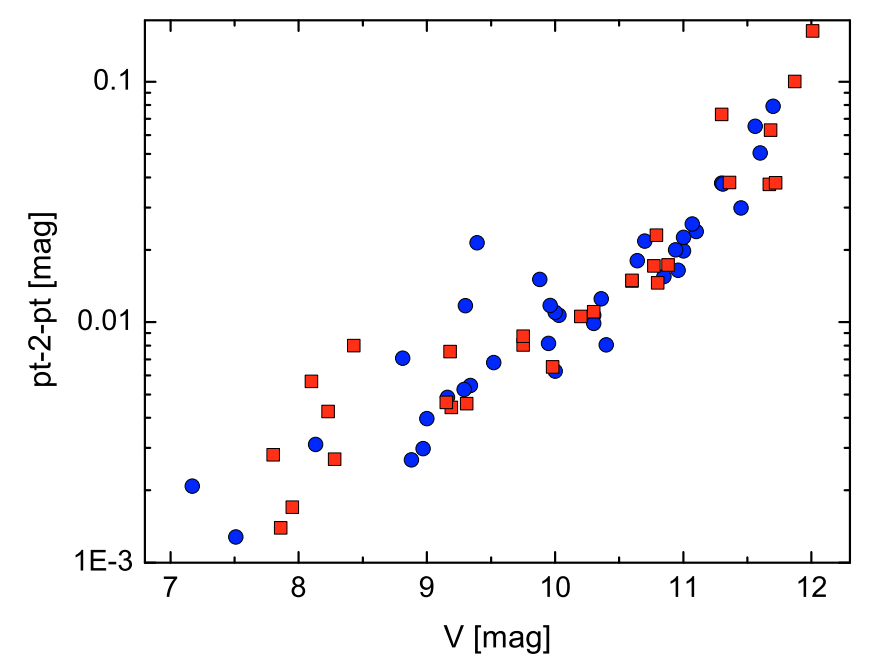

Fig. 1. Illustration of the precision of MOST guide star photometry for the NGC 2264 observations: relation of $V$ magnitude and the point-topoint scatter of the light curves (blue dots: field A; red squares: field B).

contain no variable stellar signal. If one of the stars used for the comparison light curve turns out to be variable (even at low amplitude levels) in a later stage of the analysis, the complete reduction has to be repeated omitting the variable star. A more detailed description of this method can be found in Hareter et al. (2008).

After reduction, 10531 data points were available for field A and 17698 for field B corresponding to Nyquist frequencies of $1362 \mathrm{~d}^{-1}$ and $1416 \mathrm{~d}^{-1}$, respectively. Figure 1 shows the relation between the $V$ magnitudes of the observed stars and the respective point-to-point scatter of the light curves illustrating the quality of the MOST photometry for this observing run.

SIGSPEC (Reegen 2007) was used to perform the frequency analysis of all 68 stars observed by MOST. SIGSPEC computes significance levels for amplitude spectra of time series with arbitrary time sampling. The probability density function of a given amplitude level is solved analytically and the solution includes dependencies on the frequency and phase of the signal. For the variable stars the identified (pulsational) frequencies and their significances were then verified using the Period04 software (Lenz \& Breger 2005) where Fourier and least-squares algorithms are combined. The resulting formally significant frequencies are then checked against instrumental frequencies related to the orbit of the satellite, its harmonics and $1 \mathrm{~d}^{-1}$ sidelobes within the frequency resolution (computed according to Kallinger et al. 2008c). Obviously, such peaks have to be discarded in the analysis. An example is given in Fig. 2. All frequency errors are calculated according to Kallinger et al. (2008c).

The spectral window functions of field A and field B data sets are shown in Fig. 3. The significant influence of the MOST orbital frequency of $\sim 14.2 \mathrm{~d}^{-1}$ and its multiples can clearly be seen.

Frequency analysis of the $u v b y$ data set collected at OSN was performed using the software Period04 (Lenz \& Breger 2005). The results were then verified using SIGSPEC. As usual when $u v b y$ photometry is used to investigate the pulsational content in A-F stars, the data collected in the $v$ and $b$ bands were analyzed first. Due to the temperature of the star, the fluxes in $b$ and $v$ are larger than in $u$ and $y$, hence the precisions in these two filters are better and the results are more reliable. The time-span of the data sets is 35.9 days, corresponding to a Rayleigh frequency resolution of $0.028 \mathrm{~d}^{-1}$ and an oversampling of 20 should 

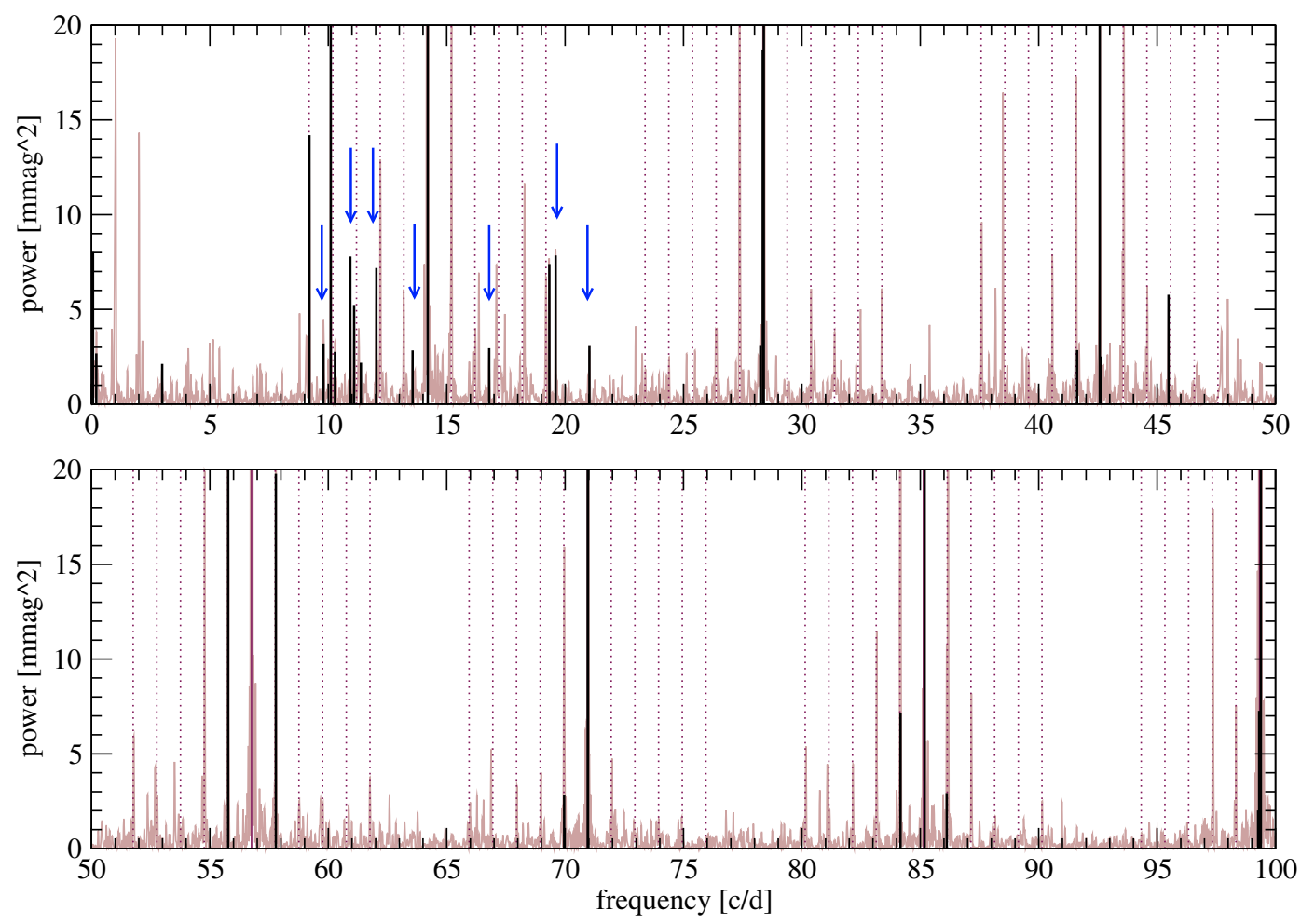

Fig. 2. Illustration of the frequency analysis using the power spectrum of HD 261230 (V1) (grey): several of the formally significant peaks (black lines) coincide with the MOST orbital frequency and its harmonics (solid lines) and the respective $1 \mathrm{~d}^{-1}$ sidelobes (dotted lines); the identified pulsation frequencies are marked with arrows.

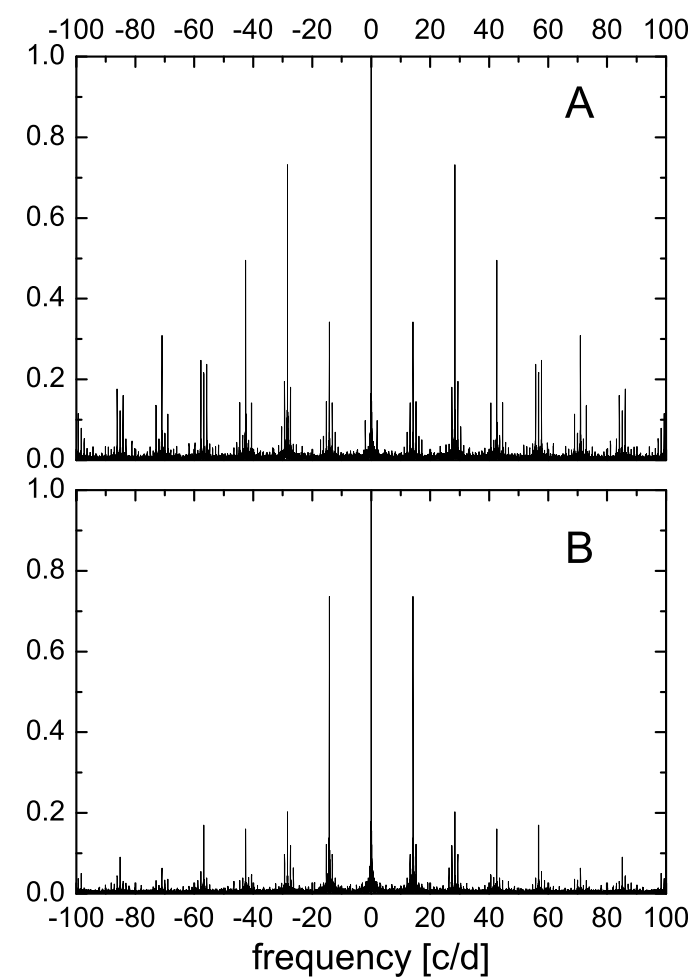

Fig. 3. Spectral windows of field A (top) and B (bottom) of the MOST observations.

correspond to a frequency spacing of $0.0014 \mathrm{~d}^{-1}$. Moreover, the time spacing between two consecutive points is about $3.5 \mathrm{~min}$ which leads to a Nyquist frequency of about $f_{\mathrm{Nyq}}=205 \mathrm{~d}^{-1}$ for the simultaneous ground-based time series.

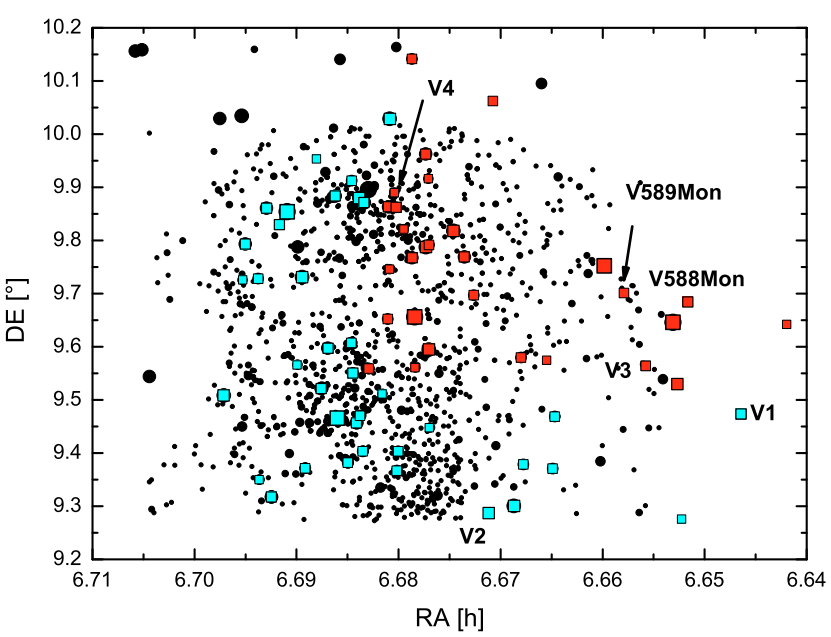

Fig. 4. Cluster chart for NGC 2264: black circles are NGC 2264 stars (taken from the WEBDA database down to $V \sim 20 \mathrm{mag}$ ) and colored squares are the stars observed by MOST (light blue: field A; red: field B), where the four new (PMS) pulsators are labelled V1 to V4 and the already known PMS pulsators are marked with their respective names, V 588 Mon and V 589 Mon. The size of the symbols corresponds to the magnitudes of the stars. The distribution of the stars in each of the two fields on the sky is caused by the L-shaped lay-out of the MOST open field (see Walker et al. 2003 for details).

\section{NGC 2264 as MOST target}

Figure 4 shows the location of stars in NGC 2264 on the sky with a field-of-view of $1^{\circ} \times 1^{\circ}$, where the symbol sizes correspond to the magnitudes of the stars. Black dots are stars in the 
Table 2. Catalog of all stars in the field A of NGC 2264 observed by MOST.

\begin{tabular}{|c|c|c|c|c|c|c|c|c|}
\hline $\begin{array}{c}\text { MOST } \\
\text { GS\# }\end{array}$ & $\begin{array}{c}\text { GSC } \\
\#\end{array}$ & $\begin{array}{c}\mathrm{HD} \\
\#\end{array}$ & $\begin{array}{c}\text { WEBDA } \\
\#\end{array}$ & $\begin{array}{c}V \\
{[\mathrm{mag}]}\end{array}$ & $\begin{array}{c}\mathrm{Sp} \\
\mathrm{Lit} / \mathrm{DDO}\end{array}$ & $\begin{array}{c}\text { Prob } \\
\%\end{array}$ & $\begin{array}{l}\text { Class } \\
\mathrm{v} / \mathrm{c}\end{array}$ & $\overline{\text { Type }}$ \\
\hline 0 & $00750-00863$ & 261230 & - & 9.39 & A7V/F2V & - & $\mathrm{v}$ & $\mathrm{V} 1$, new PMS $\delta$ Scu \\
\hline 1 & 00746-01829 & - & - & 12.0 & $\mathrm{~F} 0 /-$ & - & $\mathrm{c}$ & \\
\hline 2 & 00746-01755 & 261586 & 31 & 10.4 & A7-A3III/- & - & $\mathrm{c}$ & \\
\hline 3 & 00750-01619 & 261585 & 30 & 11.0 & A0V-A6/- & - & $\mathrm{c}$ & \\
\hline 4 & 00750-00975 & 261658 & 35 & 10.3 & A7-A3V/- & - & $\mathrm{c}$ & \\
\hline 5 & 00746-01733 & 261683 & 37 & 8.1 & K5III/K2V & - & $\mathrm{v}$ & K puls \\
\hline 6 & 00746-01783 & 261711 & 39 & 11.3 & $\mathrm{~A} 2 \mathrm{~V} /-$ & - & $\mathrm{v}$ & $\mathrm{V} 2$, new PMS $\delta$ Scu \\
\hline 7 & 00750-00951 & - & 70 & 11.1 & G3/- & - & $\mathrm{c}$ & \\
\hline 8 & 00746-01748 & 261842 & 103 & 10.0 & F3V-A2V/- & - & $\mathrm{c}$ & \\
\hline 9 & 00750-01375 & - & 99 & 11.0 & F2-A7IV/- & - & $\mathrm{c}$ & \\
\hline 10 & $00750-01637$ & - & 116 & 11.6 & G1/- & - & $\mathrm{c}$ & \\
\hline 11 & 00750-01747 & 261904 & 138 & 10.3 & $\mathrm{~A} 0.5 \mathrm{~V}-\mathrm{A} 2 \mathrm{~V} /-$ & - & $\mathrm{c}$ & \\
\hline 12 & $00750-01283$ & - & 145 & 10.6 & A $0.5 \mathrm{~V}-\mathrm{A} 5 /-$ & - & $\mathrm{c}$ & \\
\hline 13 & 00750-01655 & 261903 & 152 & 9.2 & B9V-B8Vn/- & - & $\mathrm{v}$ & SPB \\
\hline 14 & 00750-01307 & 48012 & 107 & 8.8 & A-late B/- & - & $\mathrm{v}$ & SPB \\
\hline 15 & 00750-00899 & 261941 & 165 & 10.9 & $\mathrm{~A} 2 \mathrm{~V}-\mathrm{F} 0 /-$ & - & $\mathrm{c}$ & \\
\hline 16 & 00750-01671 & 261940 & 157 & 10.0 & B8V-A05V/- & - & $\mathrm{c}$ & \\
\hline 17 & 00750-01762 & 261939 & 159 & 11.0 & $\mathrm{~A} 0 \mathrm{~V}-\mathrm{F} 0 /-$ & - & $\mathrm{c}$ & \\
\hline 18 & 00750-01804 & - & 137 & 9.9 & $\mathrm{~B} 5 \mathrm{~V} /-$ & - & $\mathrm{c}$ & \\
\hline 19 & 00750-01719 & 47887 & 178 & 7.2 & B2III/- & - & $\mathrm{v}$ & Bp \\
\hline 20 & 00750-00821 & 261938 & 142 & 9.0 & $\mathrm{~B} 6 \mathrm{~V} /-$ & - & $\mathrm{v}$ & SPB \\
\hline 21 & $00750-01241$ & 261937 & 158 & 10.4 & B8V-A5/- & - & $\mathrm{v}$ & SPB \\
\hline 22 & 00750-01107 & 262013 & 187 & 9.3 & B5Vn/- & - & $\mathrm{c}$ & \\
\hline 23 & $00750-01525$ & 262014 & 193 & 10.0 & A7III-A0V/F2V & - & $\mathrm{c}$ & \\
\hline 24 & 00746-01682 & 262041 & 205 & 10.7 & A2-A5III/- & - & $\mathrm{c}$ & \\
\hline 25 & $00750-01123$ & 261969 & 179 & 9.3 & B8V-A2/- & - & $\mathrm{c}$ & \\
\hline 26 & 00750-01779 & - & 209 & 11.3 & $\mathrm{~F} 2 \mathrm{~V} /-$ & 94 & $\mathrm{c}$ & \\
\hline 27 & $00750-01707$ & - & 196 & 11.5 & F7IV/- & 0 & $\mathrm{c}$ & \\
\hline 28 & 00750-01165 & 47934 & 206 & 8.9 & B9V/- & - & $\mathrm{v}$ & EB \\
\hline 29 & 00746-01768 & 262110 & 220 & 10.0 & A7-A0V/- & - & $\mathrm{c}$ & \\
\hline 30 & 00746-01729 & - & 224 & 11.6 & F5V/- & 65 & $\mathrm{c}$ & \\
\hline 31 & 00750-01221 & 47961 & 212 & 7.5 & B2V /- & 96 & $\mathrm{v}$ & SPB \\
\hline 32 & $00750-01213$ & 262066 & 215 & 9.3 & $\mathrm{~A} 2-\mathrm{A} 0 \mathrm{~V} /-$ & - & $\mathrm{v}$ & inconclusive \\
\hline 33 & 00750-01073 & 262108 & 222 & 10.0 & A3V-A05Va/- & - & $\mathrm{c}$ & \\
\hline 34 & $00750-01754$ & 262109 & 223 & 10.9 & F5-A7V/- & - & $\mathrm{v}$ & $\gamma$ Dor \\
\hline 35 & $00750-01563$ & - & 228 & 11.1 & $\mathrm{~F} 2 \mathrm{~V} /-$ & 94 & $\mathrm{c}$ & \\
\hline 36 & 00750-00949 & 262138 & 226 & 9.5 & A3-A0V/- & - & $\mathrm{c}$ & \\
\hline 37 & 00750-01527 & 48055 & 231 & 9.0 & B9V-B5V/- & - & $\mathrm{c}$ & \\
\hline
\end{tabular}

Column headers are: MOST Guide Star number (MOST GS\#), Guide Star Catalog number (GSC), HD number (HD), number given in the WEBDA database (WEBDA), $V$ magnitude, spectral type (Sp) taken from the literature (Lit) and - if available - from the DDO observations (DDO), membership probability (prob), variable or constant in the MOST time series (class) and type of variability (type). A “-” indicates that no respective information is available.

field of NGC 2264 (data taken from the WEBDA database ${ }^{1}$ ) and colored squares are the 68 stars observed by MOST in the two fields (light blue: field A, red: field B). The positions of the already known pulsating PMS stars, V 588 Mon and V 589 Mon, are labelled accordingly.

From the 68 stars observed in total, 30 show clear variability in the MOST light curves and their respective amplitude spectra: 12 stars are variable in field A and 18 in field B. A complete list of the stars observed by MOST is given in Tables 2 (field A) and 3 (field B).

The part of the colour-magnitude diagram (CMD) of NGC 2264, in which the stars observed by MOST are located, is shown in Fig. 5 ( $V$ and $B-V$ values are taken from Sung et al. 1997; Flaccomio et al. 1999; Rebull et al. 2002; Park et al. 2000). The instability strip (dashed lines in Fig. 5) is a transformation of the classical $\delta$ Scuti instability strip given by

\footnotetext{
1 The WEBDA database can be found at http: //www . univie.ac.at/webda
}

Rodriguez \& Breger (2001; for details see Zwintz 2008). Constant stars are given as black and variable stars as red filled circles. As the focus of the MOST observations of NGC 2264 was the detection of new pulsating PMS stars, the respective stars are discussed in detail. The other variable stars in the field are listed here for completeness, their detailed investigations will be subjects of future papers.

\section{Pulsating PMS stars in NGC 2264}

Apart from the already known pulsating PMS stars in NGC 2264, V 588 Mon and V 589 Mon, three new PMS pulsators (V2-V4) and a fourth pulsating star (V1) with unlikely cluster membership were discovered. Their positions within the field of the cluster are marked with V1 to V4 in Fig. 4.

Classification spectra for two of the four stars, HD 261230 (V1) and HD 261387 (V3), were obtained by Pribulla using the slit spectrograph in the Cassegrain focus of the $1.88 \mathrm{~m}$ telescope of the David Dunlap Observatory (DDO). 
Table 3. Catalog of all stars in the field B of NGC 2264 observed by MOST.

\begin{tabular}{|c|c|c|c|c|c|c|c|c|}
\hline $\begin{array}{c}\text { MOST } \\
\text { GS\# }\end{array}$ & $\begin{array}{c}\text { GSC } \\
\#\end{array}$ & $\begin{array}{c}\text { HD } \\
\#\end{array}$ & $\begin{array}{c}\text { WEBDA } \\
\#\end{array}$ & $\begin{array}{c}V \\
{[\mathrm{mag}]}\end{array}$ & $\begin{array}{c}\mathrm{Sp} \\
\mathrm{Lit} / \mathrm{DDO}\end{array}$ & $\begin{array}{c}\text { Prob } \\
\%\end{array}$ & $\begin{array}{c}\text { Class } \\
\text { v/c }\end{array}$ & 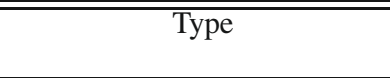 \\
\hline 0 & $00737-00304$ & 261054 & - & 9.8 & $\mathrm{~B} 3 \mathrm{e} /-$ & - & $\mathrm{V}$ & SPB \\
\hline 1 & 00750-01397 & - & - & 11.3 & $\mathrm{~B} 9 \mathrm{~V} /-$ & - & $\mathrm{v}$ & SPB \\
\hline 2 & 00750-01243 & 261331 & 2 & 9.8 & A7III-IV/- & - & $\mathrm{v}$ & V 588 Mon, known PMS $\delta$ Scu \\
\hline 3 & 00750-01713 & 261355 & 6 & 8.2 & $\mathrm{~F} 5-\mathrm{A} 0 / \mathrm{F} 2 \mathrm{~V}$ & - & $\mathrm{c}$ & \\
\hline 4 & 00750-01119 & 47469 & 7 & 7.8 & B5V-B3V/- & - & $\mathrm{v}$ & SPB \\
\hline 5 & 00750-01597 & 261387 & - & 10.6 & $\mathrm{~A} 2 \mathrm{~V} / \mathrm{A} 1 \mathrm{~V}$ & - & $\mathrm{v}$ & $\mathrm{V} 3$, new PMS $\delta$ Scu \\
\hline 6 & 00750-01820 & 261446 & 20 & 10.3 & F2III/- & - & $\mathrm{v}$ & V 589 Mon, known PMS $\delta$ Scu \\
\hline 7 & 00750-01471 & 47554 & 25 & 7.9 & $\mathrm{~F} 2 \mathrm{~V} / \mathrm{F} 2 \mathrm{~V}$ & _- & $\mathrm{v}$ & rotation? \\
\hline 8 & 00750-01039 & - & 33 & 11.7 & $\mathrm{~K} 1 \mathrm{~V} /-$ & 98 & $\mathrm{c}$ & \\
\hline 9 & 00750-01083 & 261657 & 36 & 10.9 & B9V-F0V/- & - & $\mathrm{c}$ & \\
\hline 10 & 00750-01645 & - & - & - & $-1-$ & - & $\mathrm{c}$ & \\
\hline 11 & 00750-01743 & 261737 & 43 & 10.6 & $\mathrm{~A} 7 \mathrm{~V} /-$ & - & $\mathrm{c}$ & \\
\hline 12 & 00750-01197 & 261736 & 46 & 9.2 & A3V-A5III/- & - & $\mathrm{c}$ & \\
\hline 13 & 00750-01387 & 47732 & 50 & 8.1 & B3Vnn/- & - & $\mathrm{v}$ & EB \\
\hline 14 & 00750-01163 & 261783 & 69 & 8.3 & K3II-III/K2V & - & $\mathrm{v}$ & rotation \\
\hline 15 & 00750-01825 & - & 67 & 10.8 & $\mathrm{~B} 2 \mathrm{~V} /-$ & - & $\mathrm{v}$ & EB \\
\hline 16 & 00750-01065 & - & 84 & 12.0 & G0V/- & 98 & $\mathrm{c}$ & \\
\hline 17 & 00750-01635 & 47755 & 74 & 8.4 & B9V-B3V/- & - & $\mathrm{v}$ & EB \\
\hline 18 & $00750-01425$ & 47777 & 83 & 7.9 & B3V-B2IV/- & - & $\mathrm{v}$ & Bp \\
\hline 19 & 00750-01673 & - & 68 & 11.7 & G0/- & 93 & $\mathrm{v}$ & rotation \\
\hline 20 & 00750-00879 & 261782 & 73 & 9.3 & G5IIIp/- & - & $\mathrm{c}$ & \\
\hline 21 & 00750-01778 & 261810 & 88 & 9.2 & B8V-B5V/- & - & $\mathrm{v}$ & SPB \\
\hline 22 & 00750-01745 & - & 92 & 11.7 & K0IV/- & - & $\mathrm{v}$ & rotation \\
\hline 23 & 00750-01505 & 261879 & 112 & 10.8 & A0V-A7III/- & - & $\mathrm{c}$ & \\
\hline 24 & 00750-01838 & - & 108 & 11.9 & G0/- & 95 & $\mathrm{c}$ & \\
\hline 25 & 00750-01549 & 261841 & 100 & 10.0 & A2IV/- & - & $\mathrm{v}$ & inconclusive \\
\hline 26 & 00750-00937 & 261809 & 87 & 10.8 & A4-A3V/- & - & $\mathrm{c}$ & \\
\hline 27 & $00750-01183$ & - & 104 & 11.4 & A5IV/- & 96 & $\mathrm{v}$ & V4, new PMS $\delta$ Scu \\
\hline 28 & 00750-01876 & 261878 & 109 & 9.2 & $\mathrm{~A} 0-\mathrm{B} 5 \mathrm{~V} /-$ & _- & $\mathrm{v}$ & SPB \\
\hline 29 & 00750-01767 & 261902 & 132 & 10.2 & $\mathrm{~B} 8 \mathrm{~V} /-$ & - & $\mathrm{c}$ & \\
\hline
\end{tabular}

Column headers are: MOST Guide Star number (MOST GS\#), Guide Star Catalog number (GSC), HD number (HD), number given in the WEBDA database (WEBDA), $V$ magnitude, spectral type (Sp) taken from the literature (Lit) and - if available - from the DDO observations (DDO), membership probability (prob), variable or constant in the MOST time series (class) and type of variability (type). A "-" indicates that no respective information is available.

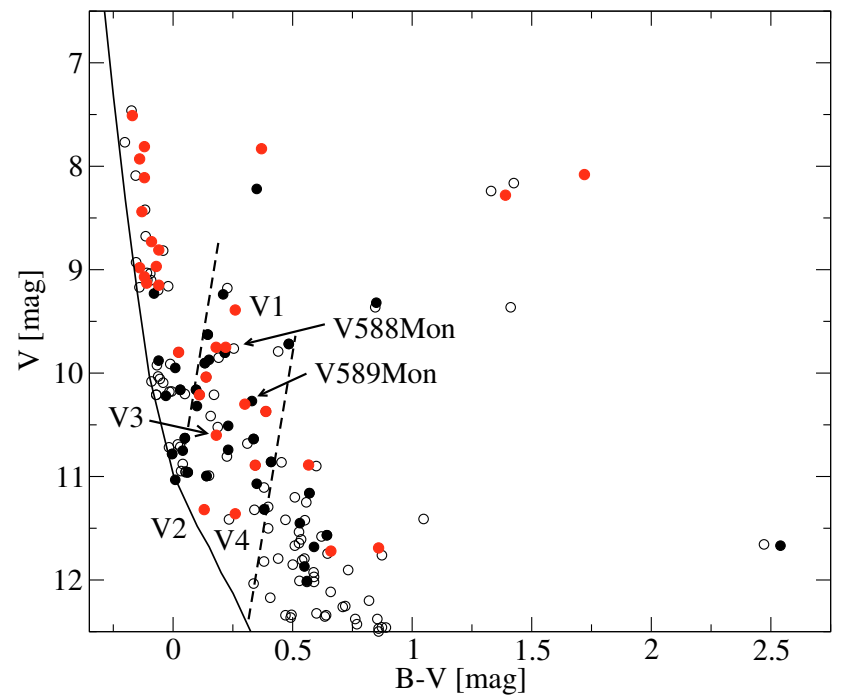

Fig. 5. Color-magnitude diagram for NGC 2264: open circles are NGC 2264 stars, filled black circles are the 38 constant stars and filled red circles are the 30 variable stars. The location of the classical instability strip is indicated with dashed lines and the ZAMS (taken from Schmidt-Kaler 1965) is marked with a solid line.

The observations were part of a program of groundbased, supportive spectroscopy for MOST Guide Stars

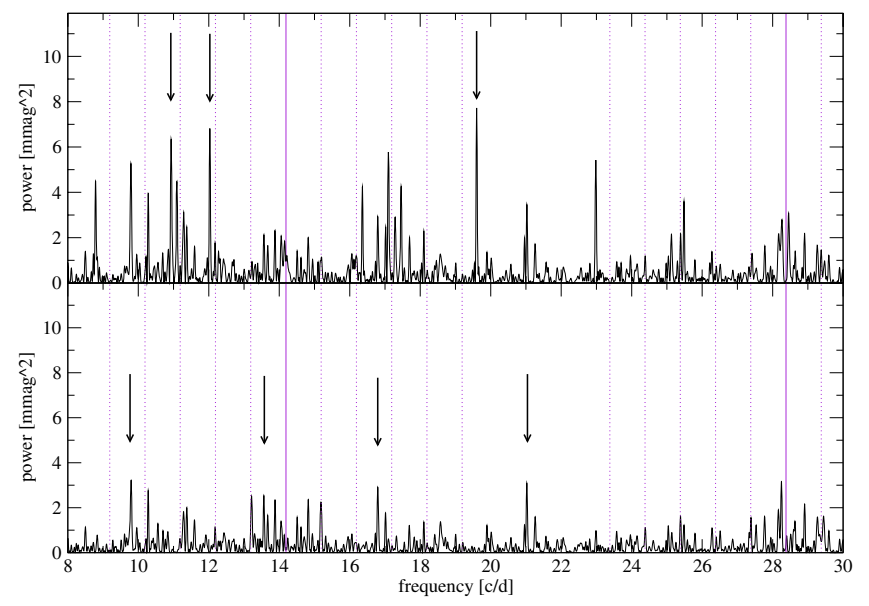

Fig. 6. Zoom into the power spectrum of HD 261230 (V1) where the identified first three (top panel) and - after prewhitening - additional four (bottom panel) pulsation frequencies are marked with arrows. Solid lines mark the multiples of the MOST orbital frequency, dotted lines are the respective $1 \mathrm{~d}^{-1}$ sidelobes. Note that the complete power spectrum from 0 to $100 \mathrm{~d}^{-1}$ is given in Fig. 2.

(Pribulla et al. 2009). The spectra were taken in a window of about $240 \AA$ around the MgI triplet (5167 ̊, $5173 \AA$ and $5184 \AA$ ) with an effective resolving power of about $12000-14000$. 


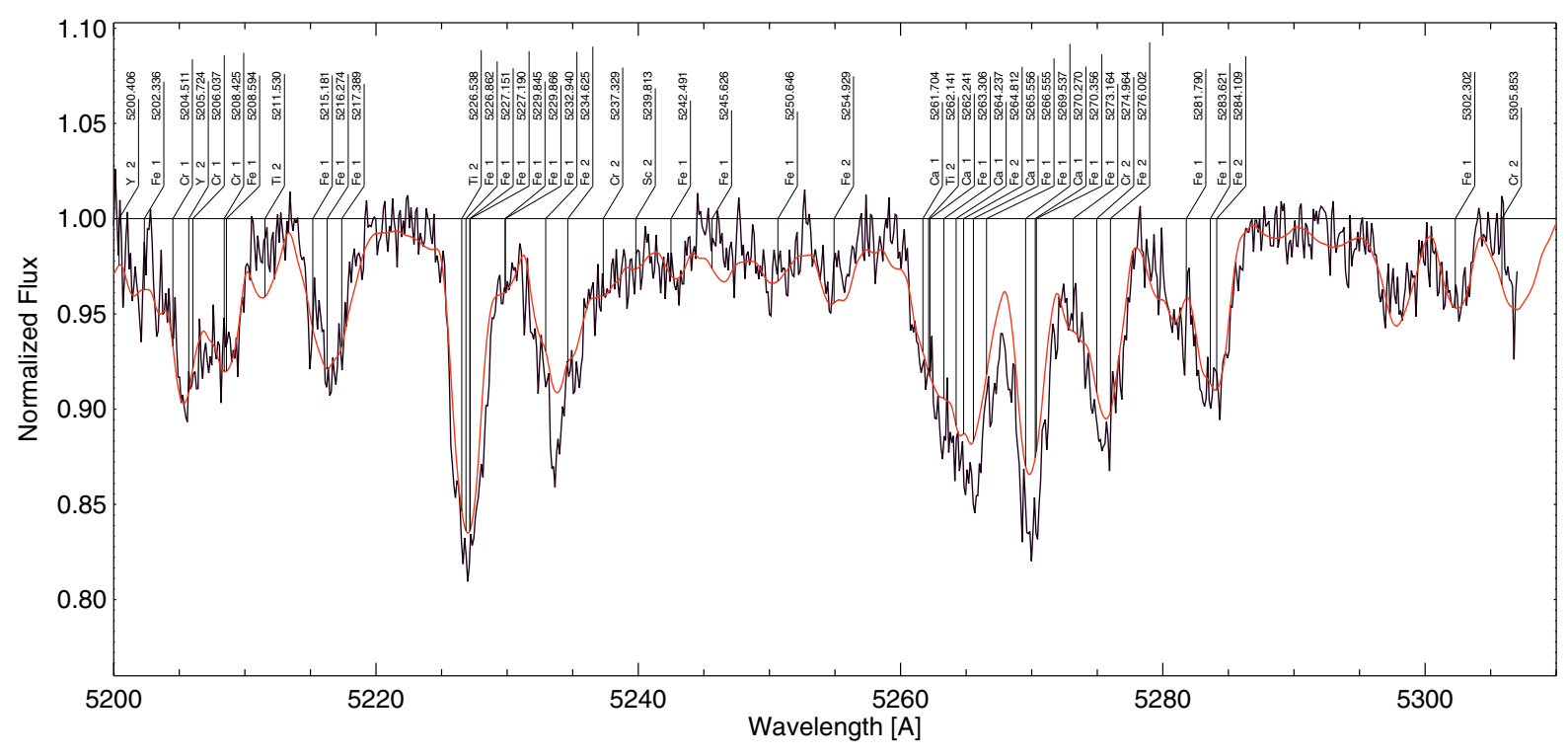

Fig. 7. Part of the classification spectrum of HD 261230 (V1; black line) obtained with the DDO $1.88 \mathrm{~m}$ telescope where the corresponding synthetic spectrum (red line) is overplotted. The labels indicate the spectral lines which contribute most to the absorption features shown in this graph.

Using these spectra, model atmospheres were calculated with LLModels8.0 (Shulyak et al. 2004) and atomic line data were extracted from the Vienna Atomic Line Database (VALD, Piskunov et al. 1995; Ryabchikova et al. 1999; Kupka et al. 1999). Synthetic spectra were calculated with synth3 (Kochukhov 2007). For detailed inspection and comparison of the synthetic spectra with the observations, the graphical IDL tool rotate (Piskunov 1992; Gruberbauer 2008, priv. comm.) was used. The results of this analysis are discussed in detail below in the respective subsections. Generally, we note differences between the spectral types reported in Pribulla et al. (2009) and the corresponding temperatures derived from our analysis. Pribulla et al. (2009) used the technique of broadening-functions (BF; Rucinski 1992 and 2002) and report that their spectral type estimates should only be treated as approximations. Our model atmosphere fits are not perfect which might be due to non-solar abundances in the atmospheres of these stars. However, the synthetic spectra based on our newly refined atmospheric parameters fit the observations significantly better than syntheses based on the previous BF spectral type estimates.

\section{1. $H D 261230(V 1)$}

For the $V=9.39 \mathrm{mag}$ star HD 261230 (GSC 00750-00863; MOST GS0A) - denoted V1 in our analysis - no membership information is available in the literature. The star is located at the edge of the field of NGC 2264 (see Fig. 4), but in the vicinity of the known cluster members V 588 Mon and V 589 Mon. Proper motion data are available, e.g. from the TYCHO Catalog (Hog et al. 1998): proper motion in right ascension (pmRA) and declination (pmDE) are given as $1.80 \pm 1.6$ and $-16.10 \pm$ $2.2 \mathrm{milliarcsec} / \mathrm{year}$ (mas/yr), respectively. Compared to the corresponding values of $-2.70 \pm 0.25$ and $-3.50 \pm 0.26 \mathrm{mas} / \mathrm{yr}$ reported for the cluster NGC 2264 (Kharchenko et al. 2001), the probability of cluster membership deduced from the proper motion measurements of HD 261230 seems to be relatively low.

In the cluster CMD (see Fig. 5) V1 lies among the other A and $\mathrm{F}$ type cluster stars which makes membership of the star to NGC 2264 not completely unlikely. For a final decision on the cluster (non-)membership of HD 261230, additional measurements are required. As the star shows a relatively rich pulsation frequency spectrum, asteroseismic modelling might also help to tackle this question (e.g., Guenther et al. 2007).

HD 261230 has a spectral type of A7V (Voroshilov et al. 1985) or F2V (Pribulla et al. 2009), hence it is located within the (PMS) $\delta$ Scuti instability strip.

We find 35 peaks to be formally significant, i.e. their significances are larger than 5.46 (Reegen 2007) which corresponds to an amplitude signal-to-noise of 4 (Breger et al. 1993; Kuschnig et al. 1997). Most of these frequencies can be related to the orbital frequency of the satellite, its harmonics and $1 \mathrm{~d}^{-1}$ sidelobes. Some peaks visible in Fig. 6 are alias frequencies due to the gaps in the observations and disappear after prewhitening the respective intrinsic frequency. Seven pulsation frequencies between 9.7 and $21.0 \mathrm{~d}^{-1}$ and amplitudes between 2.8 and $1.7 \mathrm{mmag}$ were identified (Table 6) and are marked with arrows in Fig. 6.

The classification spectrum obtained at DDO was reanalysed using the tools mentioned before. By comparison of the observations with a grid of synthetic spectra based on different model atmospheres, the atmospheric parameters were determined to be $7700 \pm 200 \mathrm{~K}$ and $\log g$ is $3.90 \pm 0.15$ (Fig. 7). Automatic fitting of the projected rotational velocity with rotate yielded $v \cdot \sin i=$ $95 \pm 5 \mathrm{~km} \mathrm{~s}^{-1}$.

\section{2. $H D 261711(\mathrm{~V} 2)$}

The $V=11.32$ mag star HD 261711 (NGC 2264 39; GSC 0074601783; MOST GS6A) - denoted V2 in our analysis - also lies in the outer regions of the cluster. Proper motion values are $-3.00 \pm$ $2.60 \mathrm{mas} / \mathrm{yr}$ in right ascension and $-4.20 \pm 2.40 \mathrm{mas} / \mathrm{yr}$ in declination (Hog et al. 2000). Compared to the values for the cluster NGC 2264 (see before), its membership seems likely.

With a spectral type of A2V (Skiff 2005) and a $(B-V)=$ 0.13 mag it falls in the instability region for (PMS) $\delta$ Scuti stars (see Fig. 5).

24 peaks are formally significant in the frequency analysis. Again, most of the frequencies can be attributed to the orbital frequency of the satellite, its harmonics and $1 \mathrm{~d}^{-1}$ sidelobes. Two 


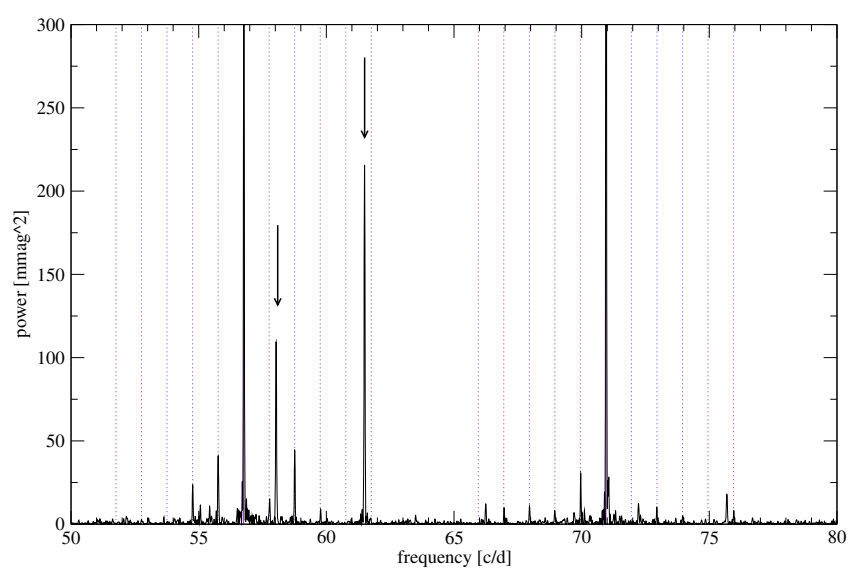

Fig. 8. Zoom into the power spectrum of HD 261711 (V2) where the two identified pulsation frequencies are marked with arrows. Solid lines mark the multiples of the MOST orbital frequency, dotted lines are the respective $1 \mathrm{~d}^{-1}$ sidelobes. Note that the complete power spectrum from 0 to $100 \mathrm{~d}^{-1}$ is only available in electronic form (Fig. 18).

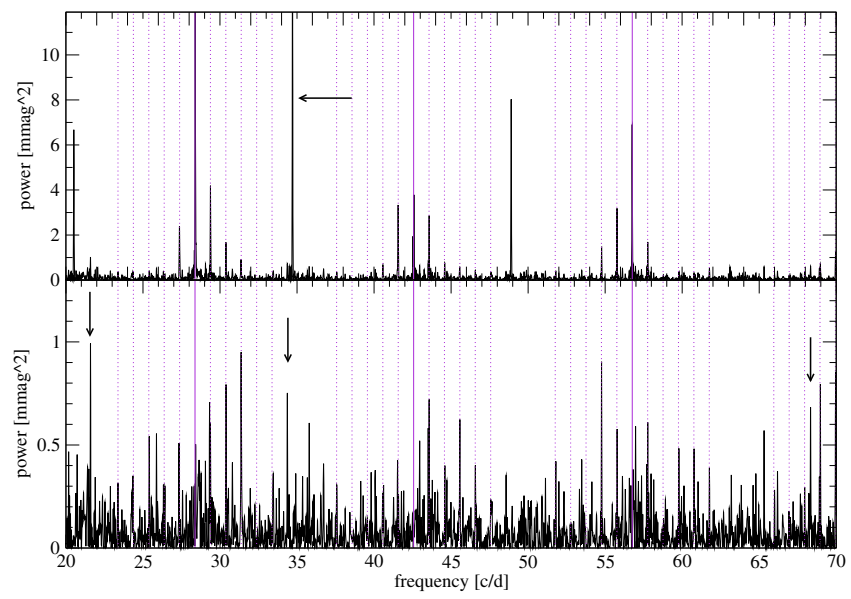

Fig. 9. Zoom into the power spectrum of HD 261387 (V3) where the first (top panel) and - after prewhitening - the additional three (lower panel) identified pulsation frequencies are marked with arrows. Solid lines mark the multiples of the MOST orbital frequency, dotted lines are the respective $1 \mathrm{~d}^{-1}$ sidelobes. Note that the complete power spectrum from 0 to $100 \mathrm{~d}^{-1}$ is only available in electronic form (Fig. 19).

peaks at $61.497 \mathrm{~d}^{-1}$ and $58.030 \mathrm{~d}^{-1}$ with amplitudes of 14.7 and $10.8 \mathrm{mmag}$, respectively, were identified to be caused by pulsation and are marked with arrows in Fig. 8.

\subsection{HD 261387 (V3)}

HD 261387 (GSC 00750-01597; MOST GS5B) - denoted V3 in our analysis - has a spectral type of A2V (Skiff 2005) or A1IV (Pribulla et al. 2009). With $V=10.60 \mathrm{mag}$ and $(B-V)=$ 0.181 mag it lies within the other $\mathrm{A}$ and $\mathrm{F}$ type stars in the cluster CMD (see Fig. 5). No membership information is available from the literature for this star. Its proper motions of $-2.1 \pm$ $2.5 \mathrm{mas} / \mathrm{yr}$ in right ascension and $-5.8 \pm 2.5 \mathrm{mas} / \mathrm{yr}$ in declination (Hog et al. 2000) suggest a probable cluster membership.

From the MOST space photometry we identified 4 out of 27 formally significant peaks to originate from pulsation (see Fig. 9 and Table 6). Note that alias frequencies due to the gaps in the observations are present, but disappear after prewhitening with the respective intrinsic frequencies.
Table 4. Amplitudes, phases, $S / N$ values and SIGSPEC significances for the only significant frequency of $34.704 \mathrm{~d}^{-1}$ derived from the OSN uvby data set of HD 261387 (V3).

\begin{tabular}{ccccr}
\hline \hline Filter & $\begin{array}{c}\text { amp } \\
{[\mathrm{mmag}]}\end{array}$ & $\begin{array}{c}\text { Phase } \\
{[\mathrm{rad}]}\end{array}$ & $S / N$ & sig \\
\hline$u$ & 3.26 & 3.682 & 4.0 & 8.4 \\
$v$ & 3.52 & 3.770 & 9.9 & 30.6 \\
$b$ & 3.04 & 3.707 & 6.5 & 5.8 \\
$y$ & 2.45 & 3.776 & 3.6 & 7.8 \\
\hline
\end{tabular}

Sigmas of the residuals in millimagnitudes are $12.2(u), 5.7(v), 7.0(b)$ and 10.7 mmag $(y)$.

Table 5. Observed phase shifts and amplitude ratios for HD 261387 (V3) where the respective errors are given in the second line.

\begin{tabular}{ccrrrr}
\hline \hline $\begin{array}{c}u-y \\
{[\mathrm{deg}]}\end{array}$ & $\begin{array}{c}v-y \\
{[\mathrm{deg}]}\end{array}$ & $\begin{array}{r}b-y \\
{[\mathrm{deg}]}\end{array}$ & $u / y$ & $v / y$ & $b / y$ \\
\hline-5.4 & -0.3 & -4.0 & 1.33 & 1.44 & 1.24 \\
22.7 & 17.0 & 18.7 & 53 & 43 & 41 \\
\hline
\end{tabular}

Table 6. Pulsation frequencies, amplitudes, SIGSPEC significances (sig) and signal-to-noise values $(S / N)$ of the four new candidate PMS pulsators obtained with SIGSPEC and PERIOD04 where the respective last digit frequency errors calculated according to Kallinger et al. (2008c) are given in parentheses.

\begin{tabular}{crrrrrr}
\hline \hline Var ID & $\#$ & \multicolumn{2}{c}{ freq } & $\begin{array}{c}\text { amp } \\
{[\mathrm{c} / \mathrm{d}]}\end{array}$ & \multicolumn{1}{c}{ sig } & $S / N$ \\
& & {$[\mathrm{mmag}]$} & & \\
\hline V1 & $f 1$ & $19.60(1)$ & $226.8(1)$ & 2.802 & 17.10 & 6.7 \\
& $f 2$ & $10.93(1)$ & $126.5(1)$ & 2.792 & 14.38 & 4.0 \\
& $f 3$ & $12.03(1)$ & $139.2(1)$ & 2.680 & 15.57 & 4.7 \\
& $f 4$ & $9.80(2)$ & $113.3(2)$ & 1.788 & 7.63 & 4.0 \\
& $f 5$ & $21.03(2)$ & $243.3(2)$ & 1.764 & 7.54 & 4.5 \\
& $f 6$ & $16.80(2)$ & $194.3(2)$ & 1.717 & 7.22 & 4.1 \\
& $f 7$ & $13.56(2)$ & $156.9(2)$ & 1.684 & 6.92 & 4.0 \\
\hline V2 & $f 1$ & $61.497(4)$ & $711.52(5)$ & 14.689 & 127.90 & 18.6 \\
& $f 2$ & $58.030(5)$ & $671.41(6)$ & 10.751 & 68.61 & 9.8 \\
\hline V3 & $f 1$ & $34.706(4)$ & $401.55(5)$ & 3.705 & 100.36 & 11.4 \\
& $f 2$ & $21.59(2)$ & $249.8(2)$ & 0.999 & 7.84 & 3.8 \\
& $f 3$ & $34.38(2)$ & $397.7(2)$ & 0.872 & 5.84 & 4.4 \\
& $f 4$ & $68.34(2)$ & $790.7(2)$ & 0.830 & 5.55 & 4.0 \\
\hline V4 & $f 1$ & $35.216(4)$ & $407.45(5)$ & 9.463 & 104.19 & 16.1 \\
& $f 2$ & $32.912(9)$ & $380.8(1)$ & 4.566 & 26.41 & 7.2 \\
& $f 3$ & $32.612(9)$ & $377.3(1)$ & 4.494 & 23.26 & 6.6 \\
\hline
\end{tabular}

The available DDO classification spectrum was compared to a set of synthetic spectra computed from a grid of model atmospheres centered on the atmospheric parameters of a mid A type star. We found that for $T_{\text {eff }}=7600 \pm 400 \mathrm{~K}$ and $\log g=3.9 \pm$ 0.4 the synthetic spectrum fits the observations best. For our comparison shown in Fig. 10 we used a projected rotational velocity $v \cdot \sin i$ of $140 \pm 10 \mathrm{~km} \mathrm{~s}^{-1}$. Since the star is fainter than HD 261230, the classification spectrum is noisier and the respective error estimates of the atmospheric parameters are larger.

For HD 261387 simultaneous ground-based Strömgren timeseries photometry was obtained with the OSN $0.9 \mathrm{~m}$ telescope (Fig. 11). The frequency analysis of the $v$ and $b$ filter light curves yielded similar features with only a single significant peak in the frequency domain typical for $\delta$ Scuti-like pulsations. It lies at $34.704 \mathrm{~d}^{-1}$ with amplitudes of 3.5 and $3.0 \mathrm{mmag}$, respectively, and confirms the frequency with the highest amplitude detected in the MOST data (i.e., $f 1$ of V3; see Table 6). Figure 12 shows the amplitude spectra of HD 261387 in the $v$ filter, before (top panel) and after (bottom panel) prewhitening with the 


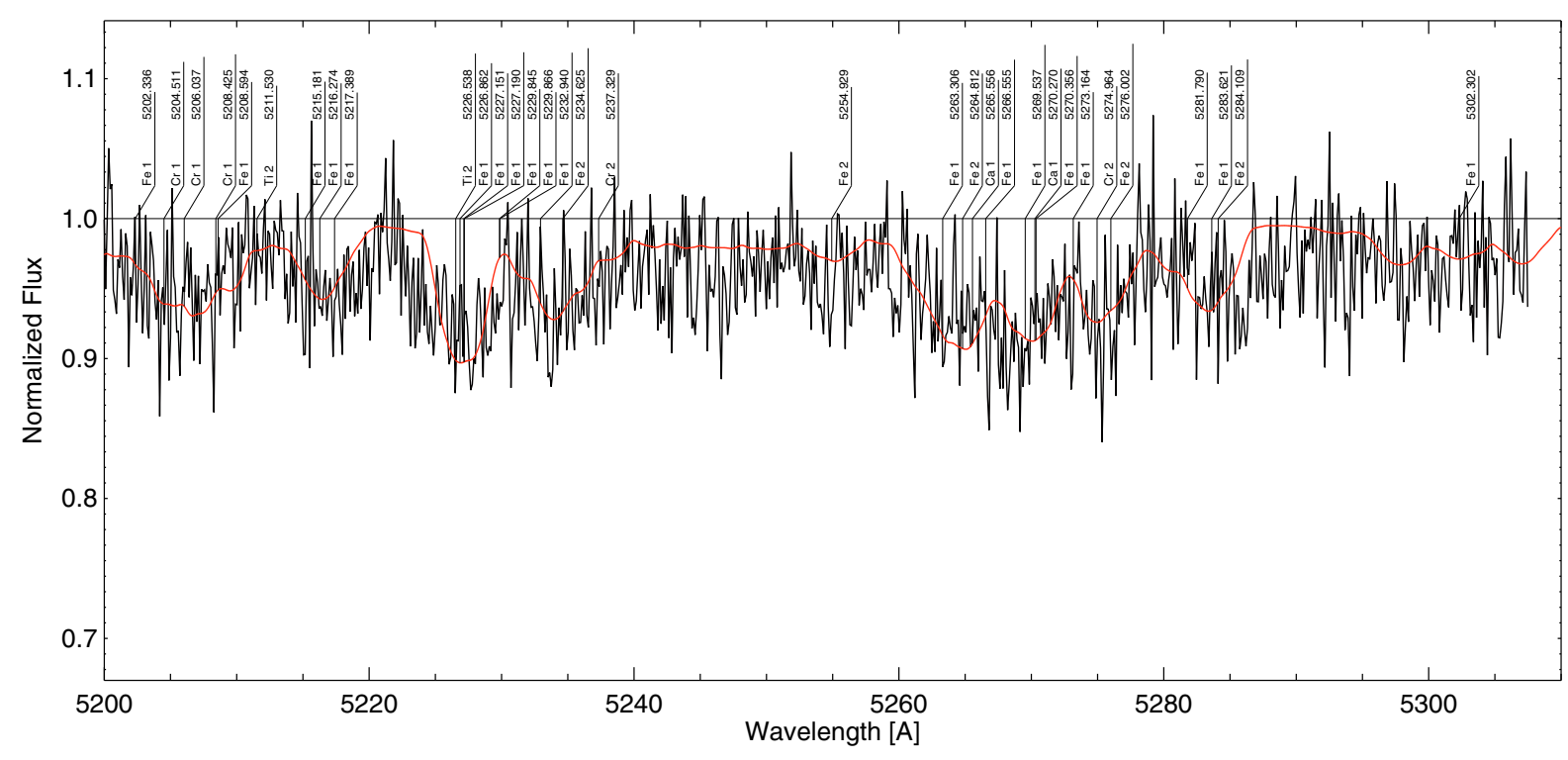

Fig. 10. Part of the classification spectrum of HD 261387 (V3; black line) obtained with the DDO $1.88 \mathrm{~m}$ telescope where the corresponding synthetic spectrum (red line) is overplotted. The labels indicate the spectral lines which contribute most to the absorption features shown in this graph.
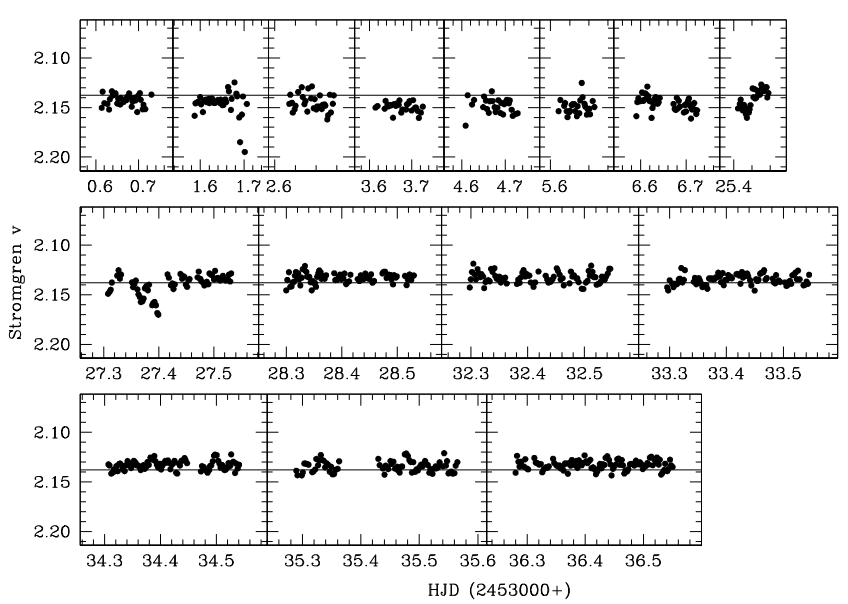

Fig. 11. Strömgren $v$ light curve for HD 261387 (V3) obtained with the OSN 0.9 m telescope.

pulsation frequency at $f 1=34.704 \mathrm{~d}^{-1}$. Table 4 shows the results of the fit for $f 1$ as applied to the light curves in all four filters. The $S / N$ values are determined following Breger et al. $(1993,1999)$ by averaging the amplitudes of the residuals over $5 \mathrm{~d}^{-1}$ boxes around the frequency under consideration. Table 5 lists the observed phase shifts and amplitude ratios determined for $f 1$. However, the error bars are too large to get any conclusion on the degree $\ell$ identification corresponding to $f 1$.

From the OSN Strömgren photometry, the standard photometry for HD 261387 was estimated by making use of the differential photometry and the standard values of the comparison stars, averaging the results. The software TempLogG ${ }^{\mathrm{TNG}}$ (Kaiser 2006) was then used, yielding the following physical parameters: $T_{\text {eff }}=8770 \pm 260 \mathrm{~K}, \log g=4.48 \pm 0.07$ and $[\mathrm{Fe} / \mathrm{H}]=+0.29 \pm$ 0.06 . The spectral type was computed as $\mathrm{A} 3 \mathrm{~V}$ and the extinction $E(b-y)=0.07 \mathrm{mag}$, which coincides with the value given by Sung et al. (1997) for the cluster. The parameters provided by the photometry are just an estimate since the calibrations in TempLogG ${ }^{\text {TNG }}$ are not optimized for PMS stars. Furthermore,

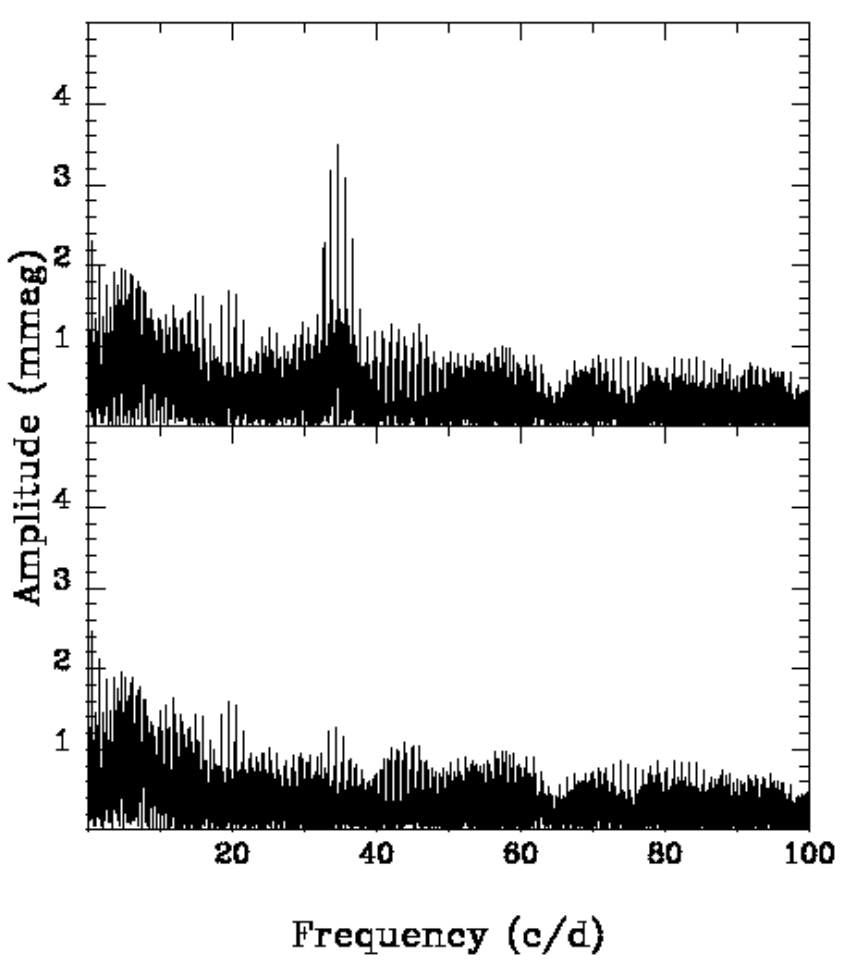

Fig. 12. Amplitude spectra of the OSN data set for HD 261387 in the $v$ filter before (top panel) and after (bottom panel) prewhitening $f_{1}$.

the photometric index providing information on the effective temperature in late-A stars is the $\beta$-index which is not available from our observations. Therefore, differences to the values derived from spectrum synthesis exist.

\subsection{NGC2264 104 (V4)}

NGC 2264104 (GSC 00750-01713; MOST GS27B) - denoted V4 in our analysis - is the only potential PMS pulsating star for which a membership probability of $96 \%$ is given 
Table 7. Main frequencies, amplitudes and total number of detected intrinsic frequencies in the respective frequency range of other variable stars.

\begin{tabular}{lllrrrrr}
\hline \hline Type & \multicolumn{1}{c}{ Star } & $\begin{array}{c}f_{\max } \\
{\left[\mathrm{d}^{-1}\right]}\end{array}$ & $\begin{array}{r}\mathrm{amp} \max \\
{[\mathrm{mmag}]}\end{array}$ & $\begin{array}{r}\text { sig } \\
\text { max }\end{array}$ & $\begin{array}{r}S / N_{\max } \\
\text { no }(f) \\
\#\end{array}$ & $\begin{array}{c}\text { range } \\
{\left[\mathrm{d}^{-1}\right]}\end{array}$ \\
\hline SPB & HD 262109 & $2.731(4)$ & 5.535 & 117.14 & 9.0 & 8 & {$[0.0,3.0]$} \\
\hline & HD 261903 & $3.216(3)$ & 2.400 & 205.16 & 13.8 & 2 & {$[3.2,3.3]$} \\
& HD 48012 & $2.788(2)$ & 6.306 & 524.35 & 17.2 & 5 & {$[2.5,3.1]$} \\
& HD 261938 & $6.503(6)$ & 0.739 & 60.46 & 11.9 & 3 & {$[0.0,6.5]$} \\
& HD 261937 & $0.089(2)$ & 12.601 & 605.54 & 9.5 & 8 & {$[0.0,0.8]$} \\
& HD 47961 & $0.058(3)$ & 0.948 & 283.65 & 5.0 & 27 & {$[0.0,3.8]$} \\
& HD 261054 & $1.976(2)$ & 5.602 & 372.47 & 8.8 & 8 & {$[0.0,2.2]$} \\
& GSC 00750-01397 & $0.239(6)$ & 12.170 & 47.89 & 5.5 & 6 & {$[0.0,0.7]$} \\
& HD 47469 & $0.054(3)$ & 0.929 & 161.16 & 4.7 & 22 & {$[0.0,3.1]$} \\
& HD 261810 & $0.071(3)$ & 1.697 & 187.67 & 9.1 & 17 & {$[0.0,7.1]$} \\
& HD 261878 & $0.147(7)$ & 1.269 & 42.74 & 3.8 & 14 & {$[0.0,2.0]$} \\
\hline Bp & HD 47887 & $0.513(1)$ & 7.092 & 1857.05 & 17.0 & 1 & {$[0.0,0.6]$} \\
& HD 47777 & $0.379(1)$ & 8.719 & 3246.02 & 16.1 & 1 & {$[0.0,0.4]$} \\
\hline spotted & HD 47554 & $1.034(4)$ & 0.551 & 140.07 & 3.6 & 3 & {$[1.0,1.6]$} \\
& HD 261783 & $0.452(2)$ & 1.894 & 561.33 & 8.5 & 7 & {$[0.0,1.0]$} \\
& NGC 2264 68 & $0.821(2)$ & 24.847 & 587.68 & 10.5 & 2 & {$[0.7,1.7]$} \\
& V 642 Mon & $1.341(1)$ & 106.470 & 2142.68 & 21.7 & 1 & {$[1.3,1.4]$} \\
\hline
\end{tabular}

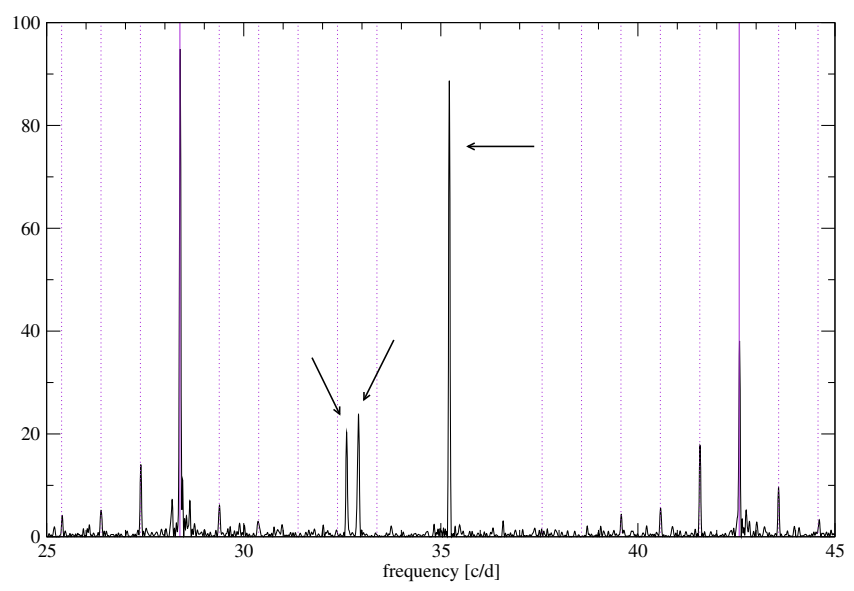

Fig. 13. Zoom into the power spectrum of NGC 2264104 (V4) where the three identified pulsation frequencies are marked with arrows. Solid lines mark the multiples of the MOST orbital frequency, dotted lines are the respective $1 \mathrm{~d}^{-1}$ sidelobes. Note that the complete power spectrum from 0 to $100 \mathrm{~d}^{-1}$ is only available in electronic form (Fig. 20).

(Vasilevskis et al. 1965). Its relative central position within the cluster (Fig. 4) and its location in the cluster CMD (Fig. 5) also indicate its membership. The spectral type is reported to be A5IV (Skiff 2005). The calculation of $T_{\text {eff }}, \log g$ and spectral type using the Strömgren photometry, e.g., from Strom et al. (1971) and the software TempLogG ${ }^{\mathrm{TNG}}$ (Kaiser 2006) yield $T_{\text {eff }}=7623 \mathrm{~K}, \log g=3.91$ and a spectral type of A8.

Using the MOST space photometry, 28 frequencies were found to be formally significant. We identified 3 relatively close peaks to be caused by pulsation (see Fig. 13 and Table 6).

\section{Other variable stars}

Numerous other variable stars were identified using the MOST time series photometry (marked with " $v$ " in Tables 2 and 3). Here, for completeness, we only give an overview of these other variable objects. Detailed investigations and discussions on the respective variabilities will be subjects of subsequent papers. Table 7 lists all stars with their identified type of variability (type), the highest frequency $\left(f_{\max }\right)$ with the respective
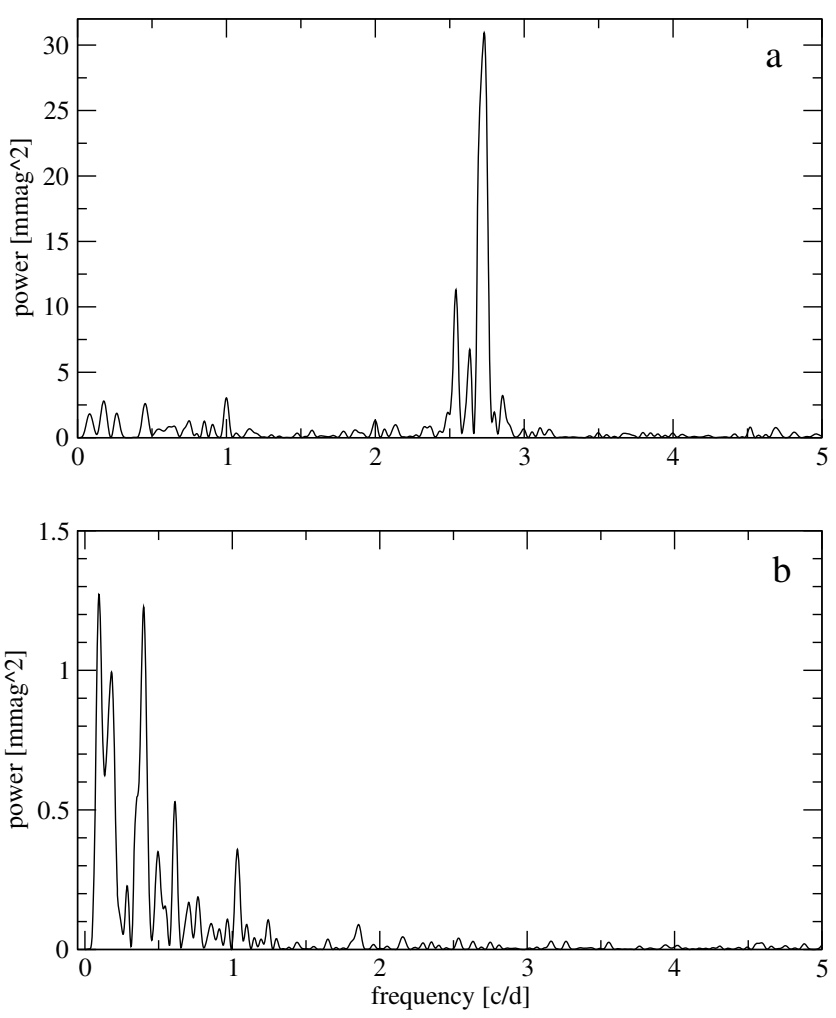

Fig. 14. Power spectra of the $\gamma$ Doradus star HD 262109 (panel a) and the pulsating K giant HD 261683 (panel b).

error in the last digit calculated according to Kallinger et al. (2008c) given in parentheses, respective amplitude $\left(\mathrm{amp}_{\max }\right)$, SIGSPEC significance ( $\left.\operatorname{sig}_{\max }\right)$, signal-to-noise value $(S / N)$ obtained with PERIOD04 and total number of detected intrinsic frequencies $(\operatorname{no}(f))$ in the respective frequency range (range).

\section{1. $\gamma$ Doradus star}

One star shows $\gamma$ Doradus type variability: HD 262109 (GSC 00750-01754, MOST GS34A) has a spectral type typical for this kind of pulsation, i.e., F5, but is not a member of the cluster as a membership probability of $0 \%$ is reported by 


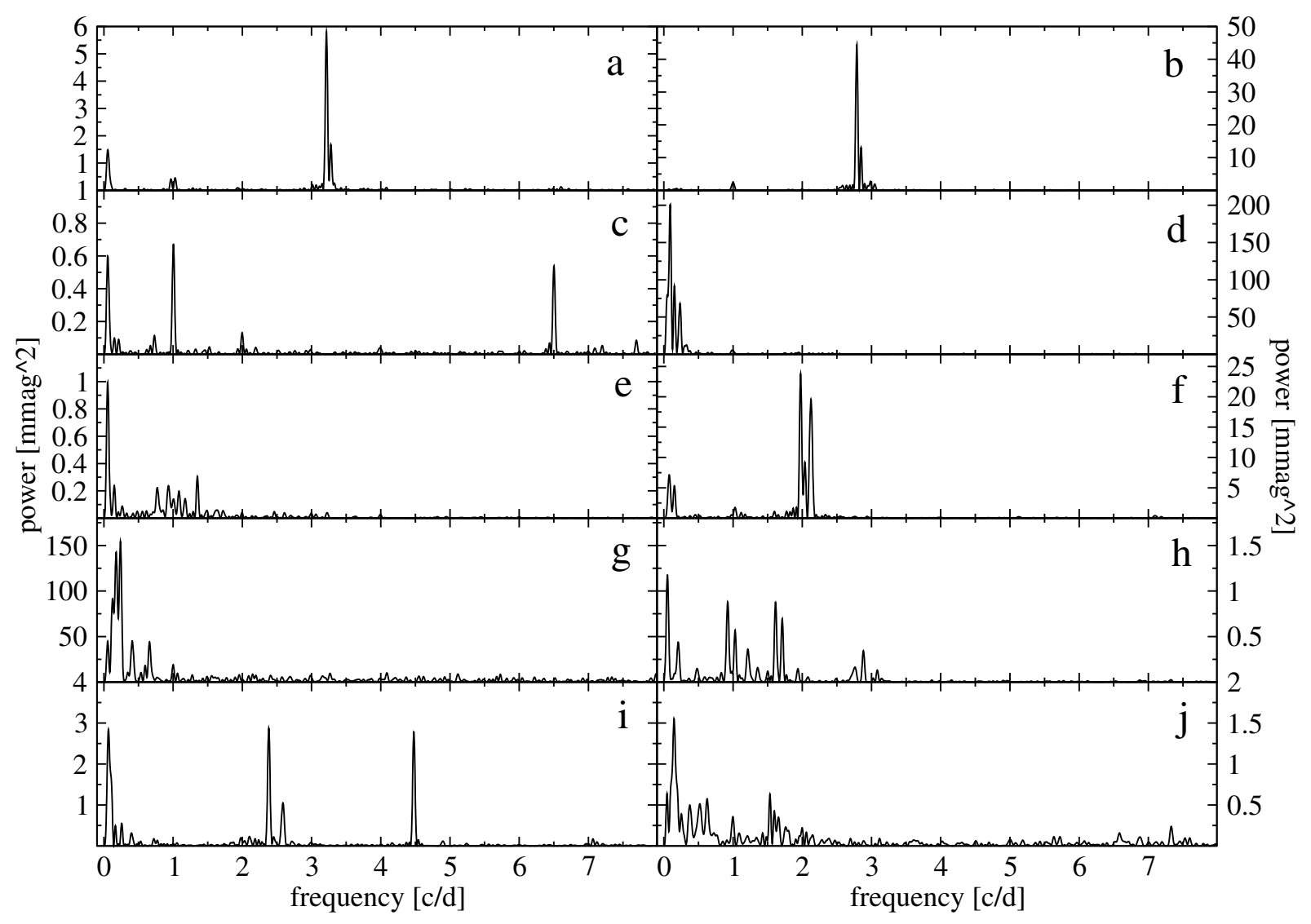

Fig. 15. Power spectra of the 10 SPB stars in the MOST field-of-view. For a detailed description see text.

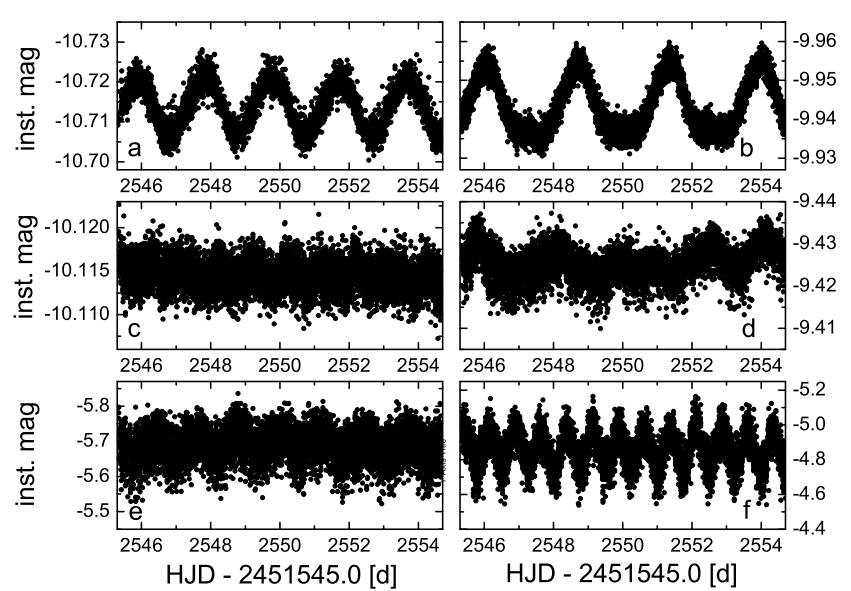

Fig. 16. Subset light curves of the five stars with rotational modulation; HD 47887 (MOST GS19A), HD 47777 (MOST GS18B), HD 47554 (MOST GS7B) HD 261783 (MOST GS14B), NGC 226468 (MOST GS19B) and V 642 Mon (MOST GS22B) in panels a to f, respectively.

Vasilevskis et al. (1965). In the MOST light curve HD 262109 shows 8 distinctive peaks between 2.5 and $2.9 \mathrm{~d}^{-1}$ with amplitudes between 5 and 1 mmag (see Fig. 14 top panel and Table 7).

\subsection{Pulsating K giant}

HD 261683 (GSC 00746-01733, MOST GS5A) has a spectral type of K5III and a $V$ magnitude of 8.13. Therefore membership of the star to NGC 2264 is very unlikely (see Fig. 5). In the

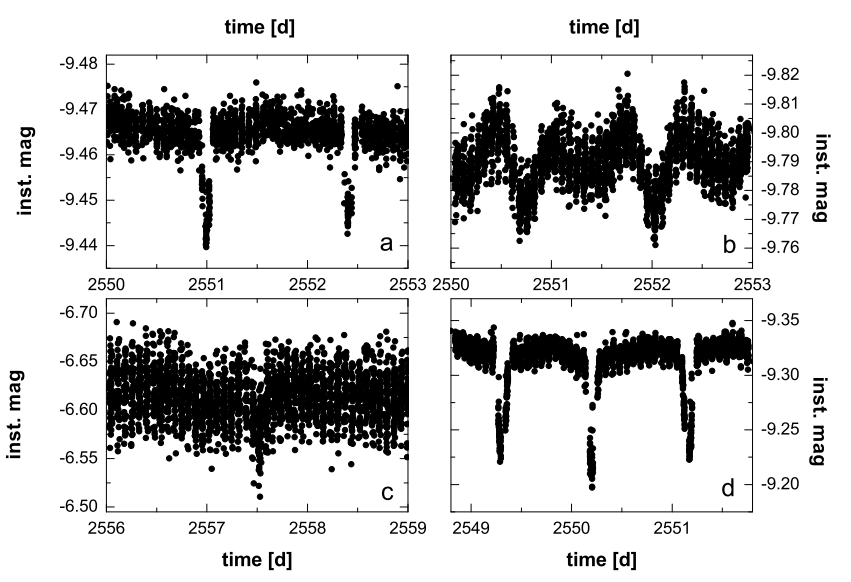

Fig. 17. Three days subset light curves of the four eclipsing binaries: HD 47934 (GSC 00750-001165, MOST GS28A), HD 47732 (GSC 00750-01387, MOST GS13B), NGC 226467 (GSC 0075001825, MOST GS15B) and HD 47755 (GSC 00750-01635, MOST GS17B) in panels a to d, respectively.

MOST photometry clear variability in the low frequency domain is present (see Fig. 14 bottom panel), which is attributed to solarlike oscillations in $\mathrm{K}$ giants as, e.g., for the case of $\epsilon$ Ophiuchi (Kallinger et al. 2008b).

\subsection{Slowly pulsating $B(S P B)$ stars}

10 stars show SPB variability in the data obtained with the MOST satellite (see Tables 2 and 3). Figure 15 panels a to 

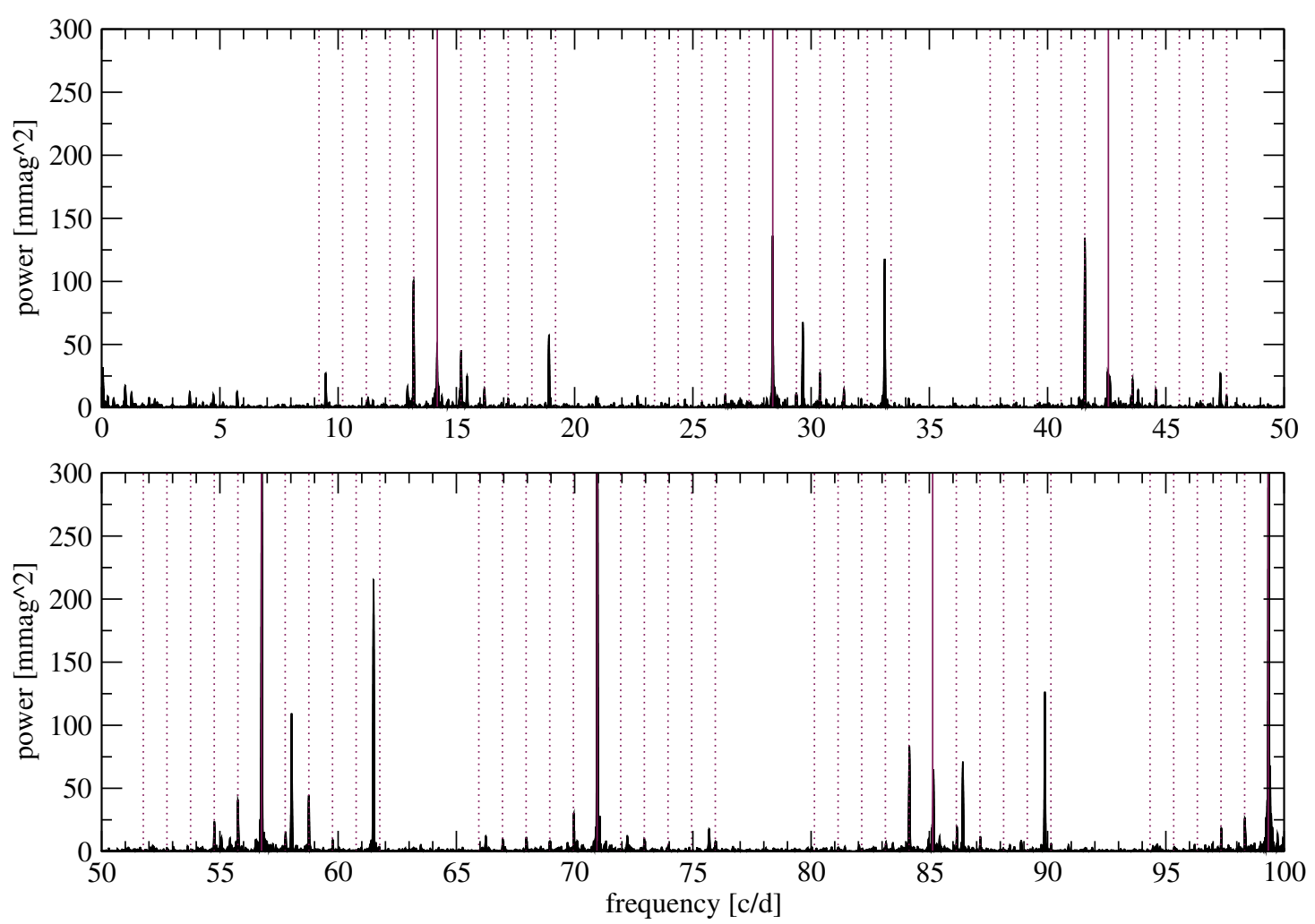

Fig. 18. Full power spectrum of HD 261711 (V2) from 0 to $100 \mathrm{~d}^{-1}$.

$\mathrm{j}$ show the respective power spectra in the following order: HD 261903 (GSC 00750-01655, MOST GS13A), HD 48012 (GSC 00750-01307, MOST GS14A), HD 261938 (GSC 0075000821, MOST GS20A), HD 261937 (GSC 00750-01241, MOST GS21A), HD 47961 (GSC 00750-1221, MOST GS31A), HD 261054 (GSC00737-00304, MOST GSOB), GSC 0075001397 (MOST GS1B), HD 47469 (GSC 00750-01119, MOST GS4B), HD 261810 (GSC 00750-01778, MOST GS21B) and HD 261878 (GSC 00750-01876, MOST GS28B). The 10 stars show frequencies between $0.05 \mathrm{~d}^{-1}$ and $6.5 \mathrm{~d}^{-1}$ corresponding to periods from 20 days to $3.7 \mathrm{~h}$ (for details see Table 7).

\subsection{Spotted stars}

Six stars with quite different spectral types show variability that is likely caused by rotational modulation. HD 47887 (GSC 00750-01719, MOST GS19A) and HD 47777 (GSC00750-01425, MOST GS18B) are known chemically peculiar stars, i.e., Bp stars. Subsets of their light curves selected for optimum visibility - are shown in panels "a" and "b" of Fig. 16 and their respective rotation frequencies are listed in Table 7. HD 47554 (GSC 00750-01471, MOST GS7B), HD 261783 (GSC 00750-01163, MOST GS14B), NGC 226468 (GSC 00750-01673, MOST GS19B) and V 642 Mon (GSC 00750-1745, MOST GS22B) have spectral types between $\mathrm{F}$ and $\mathrm{K}$ (see Tables 2 and 3). Their subset light curves are illustrated in layers $\mathrm{c}$ to $\mathrm{f}$ in Fig. 16.

\subsection{Eclipsing binaries}

Four stars in the MOST field of view were identified as eclipsing binaries. Subsets of their light curves are given in
Fig. 17 panels "a" to "d" in the following order: HD 47934 (GSC 00750-001165, MOST GS28A), HD 47732 (GSC 0075001387, MOST GS13B), NGC 226467 (GSC 00750-01825, MOST GS15B) and the previously known eclipsing binary HD 47755 (GSC 00750-01635, MOST GS17B).

\section{Summary}

High-precision time series photometry of 68 stars in the field of the young open cluster NGC 2264 was obtained using the MOST satellite. The primary goal of these observations was the search for pulsations among the PMS A and F type cluster members. Before the start of the observations, 32 objects in the MOST field-of-view were identified as potential candidates. Additionally, the two already known PMS pulsators, V 588 Mon and V 589 Mon (e.g., Kallinger et al. 2008a) were reobserved during this run. In total, 30 out of the 68 stars were found to be variable.

Three new candidate pulsating PMS stars were discovered with the MOST photometry, i.e., HD 261711, HD 261387 and NGC 2264104 . Including the two previously known pulsators in NGC 2264 this corresponds to $\sim 16 \%$ pulsational variables among the $\mathrm{A}$ and $\mathrm{F}$ type stars in the cluster. This coincides well with the percentages of pulsators in this region of the HR-diagram reported earlier for other clusters, e.g. $\sim 20 \%$ for NGC 6383 (Zwintz et al. 2005). The fourth $\delta$ Scuti type pulsator, HD 261230 (V1), is probably not a cluster member, hence its evolutionary stage remains unclear. Asteroseismic modelling of its detected pulsation frequencies might help to solve this ambiguity. Including HD 261230 (V1), the four $\delta$ Scuti-like pulsating (PMS) stars show between 2 and 7 pulsation frequencies lying in the frequency range between 9 and $62 \mathrm{~d}^{-1}$ (corresponding to periods between $2.7 \mathrm{~h}$ and $23 \mathrm{~min}$ ) and their amplitudes are at the millimagnitude level. For HD 261387 (V3) we 

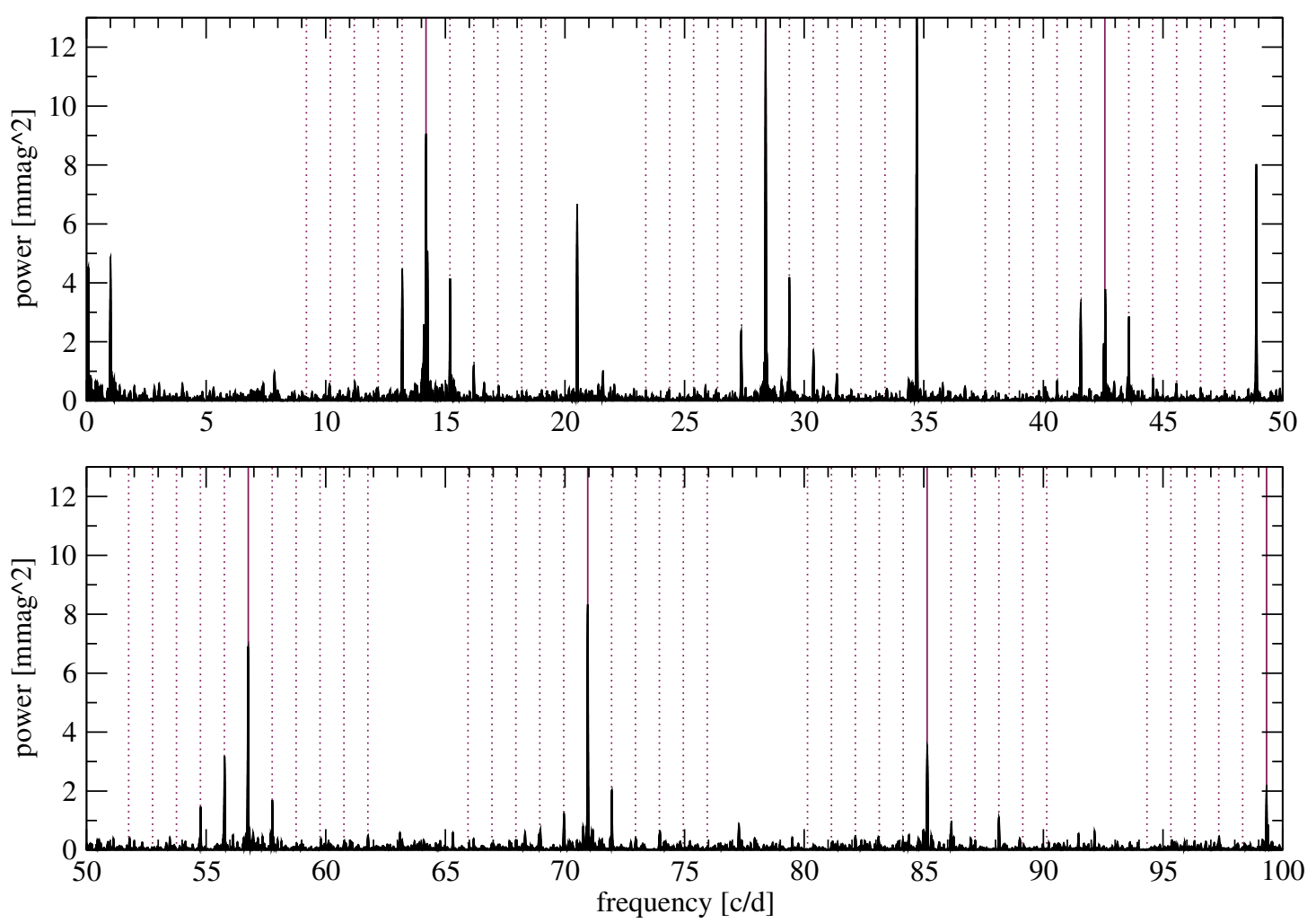

Fig. 19. Full power spectrum of HD 261387 (V3) from 0 to $100 \mathrm{~d}^{-1}$.
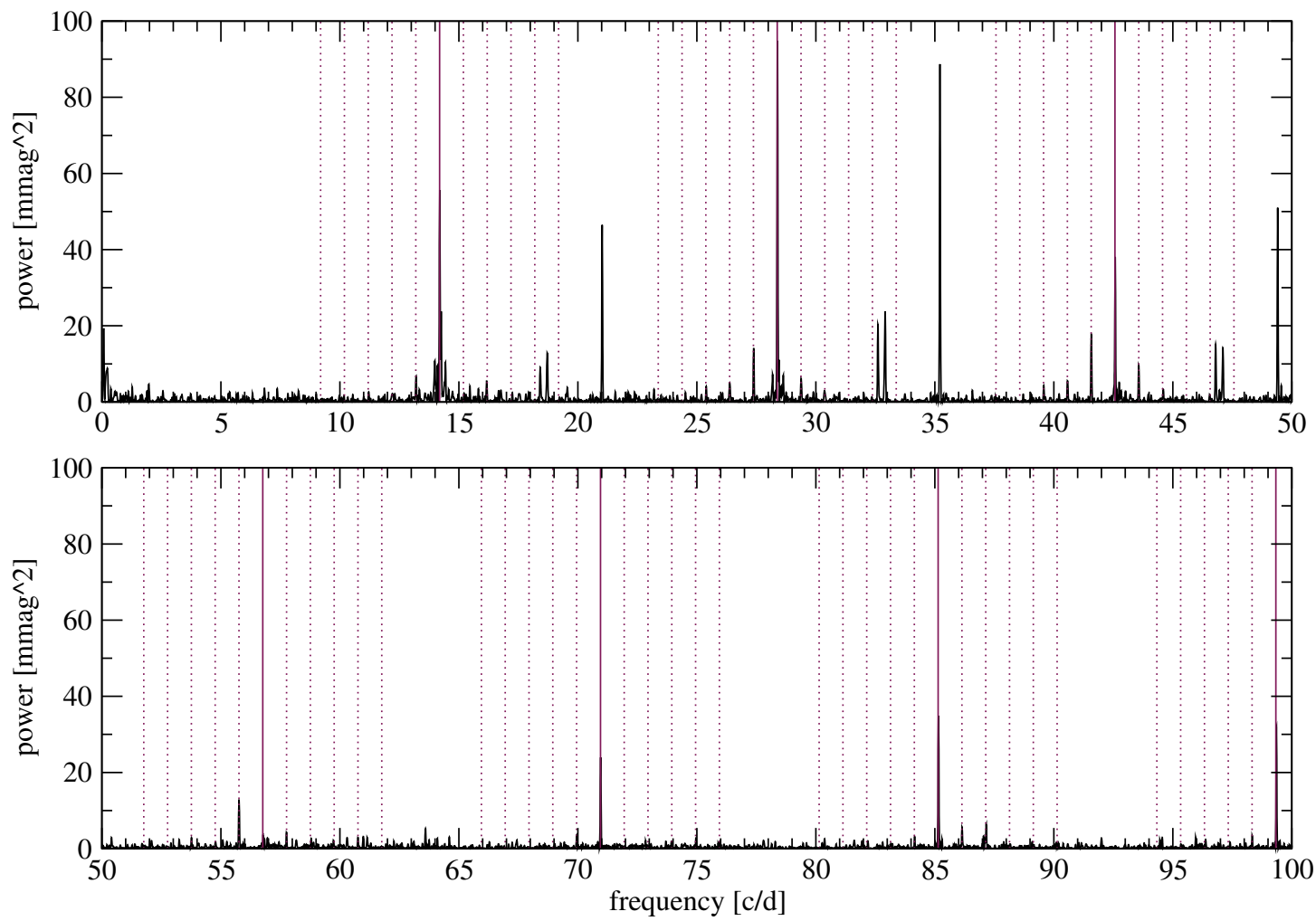

Fig. 20. Full power spectrum of NGC 2264104 (V4) from 0 to $100 \mathrm{~d}^{-1}$.

obtained Strömgren uvby photometry with the OSN $0.9 \mathrm{~m}$ telescope in parallel to the MOST observations. Combining the results confirms the existence of the main pulsation frequency at $34.706 \mathrm{~d}^{-1}\left(f_{1}\right)$.
The 34 other stars that were simultaneously observed with the MOST satellite have spectral types B, G and K. 22 of this sample show clear intrinsic variability in their light curves due to several reasons: $10 \mathrm{SPB}$ stars, $1 \gamma$ Doradus pulsator, $1 \mathrm{~K}$ 
giant oscillator, 4 eclipsing binaries, 2 Bp stars and 4 other stars with variability caused by rotational modulation. Their detailed investigations will be subjects of coming publications by other members of the team.

Acknowledgements. K.Z., M.H., N.N. and W.W.W. acknowledge support by the Austrian Fonds zur Förderung der wissenschaftlichen Forschung (K.Z.: project T335-N16; M.H., N.N. and W.W.W.: project P17890). P.J.A. acknowledges financial support from a "Ramón y Cajal" contract of the Spanish Ministry of Education and Science. The Natural Sciences and Engineering Research Council of Canada supports the research of D.B.G., J.M.M., A.F.J.M. and S.M.R.; A.F.J.M. is also supported by FQRT (Québec), and R.K. is also supported by the Canadian Space Agency. T.P. has beed funded by the Canadian Space Agency Enhancement Program (SSEP) holding a post-doctoral fellowship position at the University of Toronto and also acknowledges support from the EU in the FP6 MC ToK project MTKD-CT-2006-042514. Frequency analysis was performed using SIGSPEC (Reegen 2007) and Period04 (Lenz \& Breger 2005). This research has made use of the WEBDA database, developed by J.-C. Mermilliod (Laboratory of Astrophysics of the EPFL, Switzerland) and operated by E. Paunzen at the Institute for Astronomy of the University of Vienna. Spectroscopic observations were obtained at the David Dunlap Observatory, University of Toronto.

\section{References}

Aerts, C., De Cat, P., Kuschnig, R., et al. 2006, ApJ, 642, 165 Breger, M. 1972, ApJ, 171, 539

Breger, M., \& Pamyatnykh, A. 1998, A\&A, 332, 958

Breger, M., Stich, J., Garrido, R., et al. 1993, A\&A, 271, 482

Breger, M., Handler, G., Garrido, R., et al. 1999, A\&A, 349, 225

Finkenzeller, U., \& Mundt, R. 1984, A\&AS, 55, 109

Flaccomio, E., Micela, G., Sciortino, S., et al. 1999, A\&A, 345, 521

Guenther, D. B., Kallinger, T., Zwintz, K., Weiss, W. W., \& Tanner, J. 2007, ApJ, 671,581

Hareter, M., Reegen, P., Kuschnig, R., et al. 2008, CoAst, 156, 48

Herbig, G. H. 1960, ApJS, 4, 337

Hog, E., Kuzmin, A., Bastian, U., et al. 1998, A\&A, 335, 65

Hog, E., Fabricius, C., Makarov, V. V., et al. 2000, A\&A, 355, 27

Joy, A. H. 1942, PASP, 54, 15

Kaiser, A. 2006, ASP Conf. Ser., 349, 257

Kallinger, T., Zwintz, K., \& Weiss, W. W. 2008a, A\&A, 488, 279

Kallinger, T., Guenther, D. B., Matthews, J. M., et al. 2008b, A\&A, 478, 497

Kallinger, T., Reegen, P., \& Weiss, W. W. 2008c, A\&A, 481, 571

Kallinger, T., Zwintz, K., Guenther, D. B., et al. 2009, A\&A, in preparation

Kharchenko, N. V. 2001, Kinematics and Physics of Celestial Bodies, 17, 409

Kochukhov, O. 2007, Physics of Magnetic Stars, 109, 118
Kupka, F., Piskunov, N. E., Ryabchikova, T. A., Stempels, H. C., \& Weiss, W. W. 1999, A\&AS, 138, 119

Kuschnig, R., Weiss, W. W., Gruber, R., Bely, P. Y., \& Jenkner, H. 1997, A\&A, 328,544

Lenz, P., \& Breger, M. 2005, CoAst, 146, 5

Marconi, M., \& Palla, F. 1998, AJ, 507, L141

Palla, F., \& Stahler, S. W. 1990, ApJ, 360, L47

Palla, F., \& Stahler, S. W. 1993, ApJ, 418, 414

Park, B.-G., Sung, H., Bessel, M. S., \& Kang, Y. H. 2000, AJ, 120, 894

Pérez, M. R., Thé, P. S., \& Westerlund, B. E. 1987, PASP, 99, 1050

Piskunov, N. E. 1992, SYNTH - a code for fast spectral synthesis, in Physics and evolution of stars: Stellar magnetism, 92

Piskunov, N. E., Kupka, F., Ryabchikova, T. A., Weiss, W. W., \& Jeffery, C. S. 1995, A\&AS, 112, 525

Pribulla, T., Rucinski, S. M., Kuschnig, R., Ogloza, W., \& Pilecki, B. 2009, MNRAS, 392, 847

Rebull, L. M., Makidon, R. B., Strom, S. E., et al. 2002, AJ, 123, 1528

Reegen, P. 2007, A\&A, 467, 1353

Reegen, P., Kallinger, T., Frast, D., et al. 2006, MNRAS, 367, 1417

Rodriguez, E., \& Breger, M. 2001, A\&A, 366, 178

Rucinski, S. M. 1992, AJ, 104, 1968

Rucinski, S. M. 2002, AJ, 124, 1746

Ryabchikova, T. A., Piskunov, N. E., Stempels, H. C., Kupka, F., \& Weiss, W. W. 1999, Phis. Scr., T83, 162

Sagar, R., Piskunov, A. E., Miakutin, V. I., \& Joshi, U. C. 1986, MNRAS, 220, 383

Schmidt-Kaler 1965, in Landolt Börnstein, Numerical data and functional relationships in science and technology, group VI (Berlin: Springer Verlag), I, 284

Shulyak, D., Tsymbal, V., Ryabchikova, T., Stütz, C., \& Weiss, W. W. 2004, A\&A, 428, 993

Skiff, B. A. 2005, General Catalog of Stellar Spectral Classifications, Lowell Observatory

Strom, K. M., Strom, S. E., \& Yost, J. 1971, ApJ, 165, 479

Sung, H., Bessel, M. S., \& Lee, S.-W. 1997, AJ, 114, 2644

Sung, H., Bessel, M. S., \& Chun, M.-Y. 2004, AJ, 128, 1684

Suran, M., Goupil, M., Baglin, A., Lebreton, Y., \& Catala, C. 2001, A\&A, 372, 233

Vasilevskis, S., Sanders, W. L., \& Balz, A. G. A. 1965, AJ, 70, 797

Voroshilov, V. I., Guseva, N. G., Kalandadze, N. B., et al. 1985, in Izdatel'stvo Naukova Dumka, Kiev

Walker, M. F. 1956, ApJS, 2, 365

Walker, G., Matthews, J. M., Kuschnig, R., et al. 2003, PASP, 115, 1023

Walker, G. A. H., Kuschnig, R., Matthews, J. M., et al. 2005, ApJ, 635, 77

Zwintz, K. 2008, ApJ, 673, 1088

Zwintz, K., \& Weiss, W. W. 2006, A\&A, 457, 237

Zwintz, K., Marconi, M., Reegen, P., \& Weiss, W. W. 2005, MNRAS, 357, 345

Zwintz, K., Guenther, D. B., \& Weiss, W. W. 2007, ApJ, 655, 342 